Review

\title{
Nanotheranostics - Application and Further Development of Nanomedicine Strategies for Advanced Theranostics
}

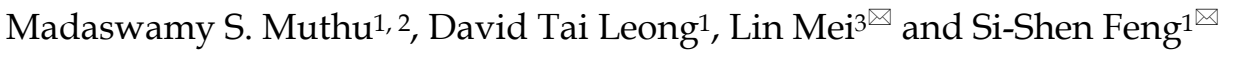 \\ 1. Department of Chemical \& Biomolecular Engineering, National University of Singapore, Blk 5 \#02-09, 4 Engineering Drive 4 , Singapore \\ 117585, Singapore. \\ 2. Department of Pharmacology, Institute of Medical Sciences, Banaras Hindu University, Varanasi 221005, India. \\ 3. Division of Life and Health Sciences, Graduate School at Shenzhen, Tsinghua University, Shenzhen 518055, China.
}

$\square$ Corresponding author: Division of Life and Health Sciences, Graduate School at Shenzhen, Tsinghua University, Shenzhen 518055, China. Tel./fax: + 86 - 75526036736; E-mail: mei.lin@sz.tsinghua.edu.cn (L. Mei). Or Department of Chemical \& Biomolecular Engineering, National University of Singapore, Blk 5 \#02-09, 4 Engineering Drive 4,Singapore 117585,Singapore. Tel.: + 65 - 6874 3835; Fax: + 65 - 6779 1936; E-mail: chefss@nus.edu.sg (Si-Shen Feng).

(C) Ivyspring International Publisher. This is an open-access article distributed under the terms of the Creative Commons License (http://creativecommons.org/ licenses/by-nc-nd/3.0/). Reproduction is permitted for personal, noncommercial use, provided that the article is in whole, unmodified, and properly cited.

Received: 2014.01.27; Accepted: 2014.03.II; Published: 2014.03.26

\begin{abstract}
Nanotheranostics is to apply and further develop nanomedicine strategies for advanced theranostics. This review summarizes the various nanocarriers developed so far in the literature for nanotheranostics, which include polymer conjugations, dendrimers, micelles, liposomes, metal and inorganic nanoparticles, carbon nanotubes, and nanoparticles of biodegradable polymers for sustained, controlled and targeted co-delivery of diagnostic and therapeutic agents for better theranostic effects with fewer side effects. The theranostic nanomedicine can achieve systemic circulation, evade host defenses and deliver the drug and diagnostic agents at the targeted site to diagnose and treat the disease at cellular and molecular level. The therapeutic and diagnostic agents are formulated in nanomedicine as a single theranostic platform, which can then be further conjugated to biological ligand for targeting. Nanotheranostics can also promote stimuli-responsive release, synergetic and combinatory therapy, siRNA co-delivery, multimodality therapies, oral delivery, delivery across the blood-brain barrier as well as escape from intracellular autophagy. The fruition of nanotheranostics will be able to provide personalized therapy with bright prognosis, which makes even the fatal diseases curable or at least treatable at the earliest stage.
\end{abstract}

Key words: Cancer nanotechnology; Drug targeting; Molecular biomaterials; Molecular imaging; Oral chemotherapy; Pharmaceutical nanotechnology.

\section{Introduction}

The word "theranostics" refers to the simultaneous integration of diagnosis and therapy [1]. Nanotheranostics is to apply and further develop nanomedicine strategies for advanced theranostics, i.e. to apply and further develop the various nanocarriers such as polymer conjugations, dendrimers, micelles, liposomes, metal and inorganic nanoparticles, carbon nanotubes, nanoparticles of biodegradable polymers for sustained, controlled and targeted co-delivery of diagnostic and therapeutic agents for better theranostic effects and fewer side effects. The purpose is to diagnose and treat the diseases at their earliest stage, when the diseases are most likely curable or at least treatable. Nanotheranostics can be promising 
even for the fatal diseases such as cancer, cardiovascular diseases and AIDS, which creates the chance to make treatment much less troublesome and prognosis bright, thus saving resources and enhancing the quality of life for the patients. Nanomedicine, defined as application of nanotechnology to diagnose, treat and prevent diseases at cellular and molecular level, can also be efficient for development of theranostic candidate to combine diagnosis and therapy simultaneously [1-3]. Advanced theranostic nanomedicine is multifunctional in nature, capable of diagnosis and delivery of therapy to the diseased cells with the help of targeting ligand and biomarkers [3-6].

Mainly for medical purposes: Theranostic nanomedicine means colloidal nanoparticles ranging in sizes from 10 to $1000 \mathrm{~nm}(1 \mu \mathrm{m})$. They consist of macromolecular materials/polymers in which the diagnostic and therapeutic agents are adsorbed, conjugated, entrapped, encapsulated for diagnosis and treatment simultaneously at cellular and molecular level [7-9].

Theranostic nanomedicine can work better than other theranostics since they have advanced capabilities in an all-in-one single platform, which include sustained/controlled release, targeted delivery, higher transport efficiency by endocytosis [9], stimulus responsive agent release (i.e., smart delivery) $[9,10]$, synergetic performance (e.g., combination therapy, siRNA co-delivery) [11], multimodality diagnosis and/or therapies and quality performances (e.g., oral delivery, escape from multi drug resistance (MDR) protein, autophagy inhibition etc) [12-17].The encapsulation of single diagnostic or therapeutic agent in nanomedicine may not have high efficacy/specificity/sensitivity for the applications. There-

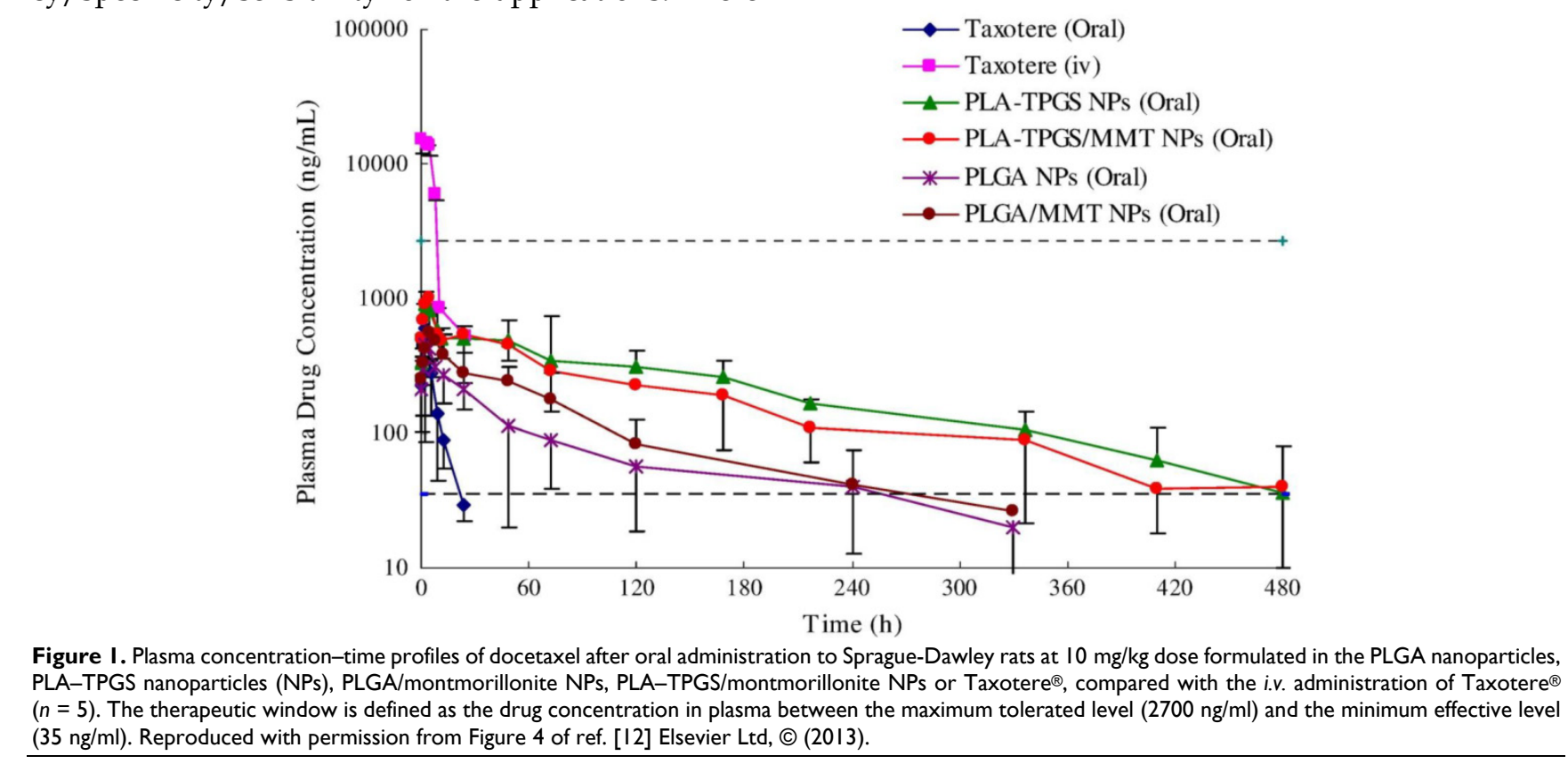

Figure I. Plasma concentration-time profiles of docetaxel after oral administration to Sprague-Dawley rats at $10 \mathrm{mg} / \mathrm{kg}$ dose formulated in the PLGA nanoparticles, PLA-TPGS nanoparticles (NPs), PLGA/montmorillonite NPs, PLA-TPGS/montmorillonite NPs or Taxotere ${ }^{\circledR}$, compared with the i.v. administration of Taxotere ${ }^{\circledR}$ $(n=5)$. The therapeutic window is defined as the drug concentration in plasma between the maximum tolerated level $(2700 \mathrm{ng} / \mathrm{ml})$ and the minimum effective level $(35 \mathrm{ng} / \mathrm{ml})$. Reproduced with permission from Figure 4 of ref. [12] Elsevier Ltd, @ (20I3). fore, multimodality nanotheranostics can be developed to make use of the advantages, which may be achieved by co-encapsulation of multiple diagnostic modes and therapeutic modes in targeting nanomedicine platforms [18]. Interestingly, siRNA can also be included in theranostic nanomedicine as inhibitor of theranostic resistance. The siRNA based theranostic nanomedicine has shown to have greatly improved the diagnosis and therapy as multimodality therapy [11]. As a novel approach, nanomedicine has also been developed for oral chemotherapy. We have reported D-a-tocopheryl polyethylene glycol 1000 succinate (TPGS) based nanomedicine for oral delivery of chemotherapeutic agents (e.g., docetaxel and paclitaxel) with enhanced oral bioavailability (Figure 1) $[12,19,20]$. Oral delivery of theranostic nanomedicine (i.e., oral theranostics) will be a task for future research, which will radically promote the practice of cancer theranostics and improve the quality of life for cancer patients, realizing the patients' dream of "chemotherapy at home" [12]. Autophagy or autophagocytosis is a catabolic process in which intracellular degradation of dysfunctional cellular components or foreign invaders occurs in lysosomes. Also, autophagy affects nanomedicine after endocytosis and its therapeutic effect by changing intracellular pharmacokinetics of nanomedicine (i.e., absorption, distribution, excretion and metabolism of nanomedicine). Therefore, autophagy inhibitors loaded in the theranostic nanomedicine can enhance the delivery of diagnostic and therapeutic agents [13]. Thus, advanced nanotheransotics has been under progress for concurrent diagnosis and therapy of diseases in high efficiency at the cellular and molecular level $[16,17]$. 
The therapeutic agents in theranostic nanomedicine also include hydrophobic organic drugs, proteins, peptides and genetic materials. In addition to therapeutic agent, diagnostic agents commonly used in theranostic nanomedicine include those for optical imaging (using fluorescent dyes or quantum dots), magnetic resonance imaging (MRI) (using superparamagnetic metals i.e., iron oxides), nuclear imaging (using radionuclides) and computed tomography (using heavy elements e.g., iodine) [3, 5, 6]. Quantum dots are semiconductor crystals that emit signal when stimulated by external energy sources. The quantum dots are popular nanomaterial among the diagnostic agents used within theranostic nanomedicine as they have significant advantages over organic dyes. Furthermore, quantum dots emit highly intense signals, larger absorption coefficients, high level of brightness and they are photostable [21, 22].

Any disease would be curable or at least treatable if it can be diagnosed at its earliest stage [8]. Hence, advanced theranostic platforms were made for sensitive diagnosis, accurate targeting, effective and controlled delivery of materials [16, 23]. Here, this review was carried out with a view to summarize the recent works on advanced theranostic nanomedicine platforms such as drug-polymer conjugates, polymeric/magnetic nanoparticles, solid lipid nanoparticles, dendrimers, liposomes, micelles, gold nanoparticles and carbon nanomaterials. The advantages of theranostic nanomedicine for the future generations include: 1) intracellular diagnosis and in vivo biodistribution 2) advanced therapy 3) therapeutic monitoring following the treatment $[24,25]$.

\section{Theranostic nanomedicine}

Theranostic nanomedicine may be defined as nanomedicine that combines diagnostics with thera- peutics [3]. The variety of nanomedicine platforms are exploited for the purposes, which are summarized in Table 1. Indeed, some nanomedicines e.g., gold nanoparticles, magnetic nanoparticles or carbon nanotubes have intrinsic diagnostic/therapeutic properties. They act as self theranostic nanomedicines or platforms. The advanced theranostic nanomedicines conjugated with targeting moiety can recognize specific target, bind and be internalized via specific mechanism e.g., receptor mediated endocytosis [26]. The nanomedicine of appropriate size and surface coating can have prolonged half-life in the circulation after intravenous (i.v.) administration [27]. It was observed that nanomedicines in the size range of 100-200 $\mathrm{nm}$ with hydrophilic surface modifications such as polyethylene glycol (PEG) and vitamin E TPGS are commonly used with best effects for theranostic applications [19, 27-30]. For example, we have reported docetaxel loaded poly-lactic acid-D-a-tocopheryl polyethylene glycol 1000 succinate (PLA-TPGS) nanoparticles having hydrophilic surface modification which can achieve a $360 \mathrm{~h}$ effective chemotherapy in comparison with only $22 \mathrm{~h}$ for Taxotere ${ }^{\circledR}$ after i.v. administration (Figure 2) [31]. In recent work, we investigated the effects of the PEG chain length of the TPGS on the cellular uptake efficiency and found that shortest PEG tethering chain length, i.e. PEG 1000, could have the highest cellular uptake of the nanoparticles due to their highest surface energy to overcome the bending energy needed for the detached piece of cell membrane bilayer to rap the nanomedicine into the biological cells by the mechanism of endocytosis [32]. The interesting preliminary work done by our group suggested the potential use of TPGS 1000 for the development of TPGS based theranostic nanomedicines to achieve desired effects.

Table I. Some advanced theranostic nanomedicine platforms under progress for simultaneous integration of diagnosis and therapy.

\begin{tabular}{|c|c|c|c|c|c|c|c|}
\hline $\begin{array}{l}\text { Type of theranostic } \\
\text { nanomedicine }\end{array}$ & Material (s) & Therapeutic agent & Diagnostic agent & Size & $\begin{array}{l}\text { Targeting } \\
\text { agent }\end{array}$ & Advancement & $\begin{array}{l}\text { Refer- } \\
\text { ence }\end{array}$ \\
\hline Drug-polymer conjugates & HPMA & $64 \mathrm{Cu}$ & $64 \mathrm{Cu}$ & N.A. & RGD & $\begin{array}{l}\text { Cancer imaging and radi- } \\
\text { ochemo-therapy }\end{array}$ & [39] \\
\hline $\begin{array}{l}\text { Polymeric } \\
\text { nanoparticles }\end{array}$ & PLA-TPGS & Docetaxel & Quantum dots & $\sim 250 \mathrm{~nm}$ & Folic acid & $\begin{array}{l}\text { Co-delivery of docetaxel and } \\
\text { quantum dots }\end{array}$ & [59] \\
\hline Solid lipid & Low-density & Paclitaxel/ & Quantum dots & $\sim 130 \mathrm{~nm}$ & cRGD & & {$[66]$} \\
\hline nanoparticles & $\begin{array}{l}\text { lipoprotein, } \\
\text { Cholesterol }\end{array}$ & siRNA & & & & $\begin{array}{l}\text { Multimodal } \\
\text { therapy }\end{array}$ & \\
\hline Dendrimers & $\begin{array}{l}\text { Polypro- } \\
\text { pyleni-mine }\end{array}$ & Phthalocyanines & $\begin{array}{l}\text { Phthalo } \\
\text { cyanines }\end{array}$ & $\sim 62 \mathrm{~nm}$ & LHRH & Delivery of single theranostic agent & [75] \\
\hline Liposomes & $\begin{array}{l}\text { TPGS, } \\
\text { Phospholipids, } \\
\text { Cholesterol }\end{array}$ & Docetaxel & Quantum dots & $\sim 210 \mathrm{~nm}$ & Folic acid & $\begin{array}{l}\text { Co-delivery of docetaxel and } \\
\text { quantum dots }\end{array}$ & {$[88]$} \\
\hline Micelles & TPGS & $\begin{array}{l}\text { Iron oxide } \\
\text { nanoparticles }\end{array}$ & $\begin{array}{l}\text { Iron oxide na- } \\
\text { noparticles }\end{array}$ & $\sim 178 \mathrm{~nm}$ & Passive & Delivery of single theranostic agent & {$[108]$} \\
\hline $\begin{array}{l}\text { Gold } \\
\text { nanoparticles }\end{array}$ & Gold nanoparticles & DOX & $\begin{array}{l}\text { Gold } \\
\text { nanoparticles }\end{array}$ & $\sim 55 \mathrm{~nm}$ & $\begin{array}{l}\text { CPLGLAG } \\
\text { G peptide }\end{array}$ & Stimulus responsive drug release & [120] \\
\hline $\begin{array}{l}\text { Carbon } \\
\text { nanomaterials }\end{array}$ & SWCNTs & Intrinsic property & $\begin{array}{l}\text { Intrinsic prop- } \\
\text { erty }\end{array}$ & $\begin{array}{l}\text { Length of } \\
\sim 140 \mathrm{~nm}\end{array}$ & Passive & $\begin{array}{l}\text { Self photolumines-cent and pho- } \\
\text { tothermal property }\end{array}$ & [131] \\
\hline
\end{tabular}




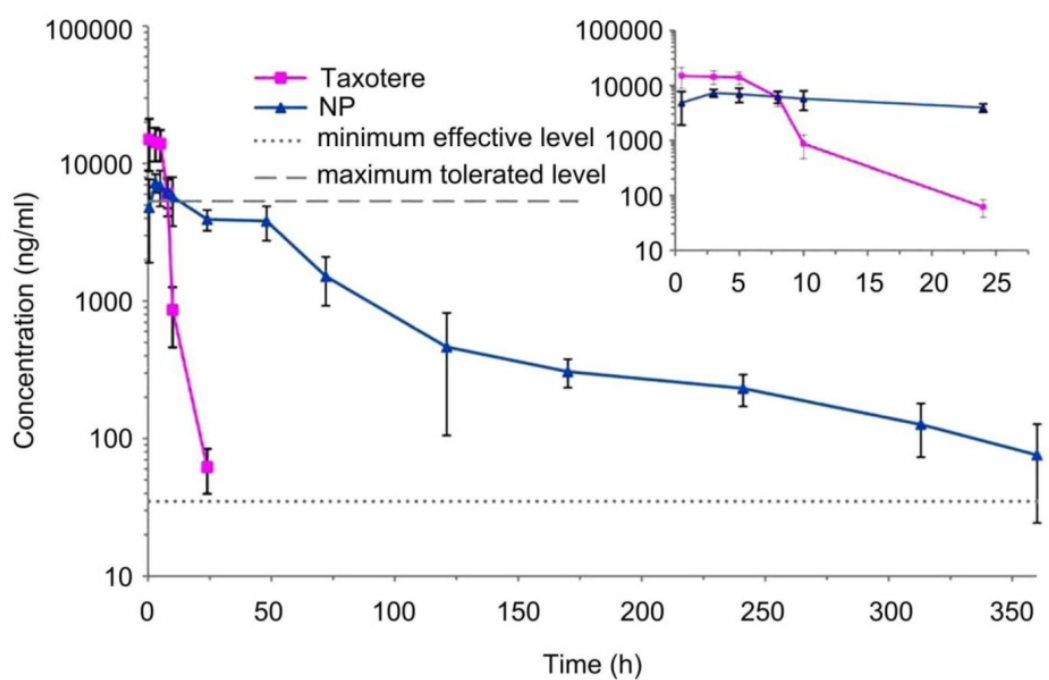

Figure 2. In vivo pharmacokinetics profiles of docetaxel plasma concentration vs. time after i.v. administration of Taxotere ${ }^{\circledR}$ and the TPGS-emulsified PLA-TPGS NPs formulation using Sprague-Dawley rats at the same docetaxel dose of $10 \mathrm{mg} / \mathrm{kg}(n=5)$. Reproduced with permission from Figure 5 of ref. [3I] Bentham Science Publishers Ltd, (C) (20I0).

\section{I. Drug-polymer conjugates}

Drug-polymer conjugates are prepared by covalent interactions using different chemical pathways that mainly depend on the functional groups of drug and polymeric carrier involved in the chemical conjugations. The two major types of conjugates include protein conjugates and drug conjugates with appropriate polymers [33]. The most successful polymer for theranostic drug-polymer conjugate is N-(2-hydroxypropyl) methacrylamide (HPMA) [33]. The common methods for functionalization of HPMA copolymers with diagnostic and therapeutic agents are chemical conjugation and copolymerization [33, 34]. HPMA based conjugates are used for theranostics since they are stable, non-toxic and biocompatible for in vivo applications $[33,35,36]$. There are many studies showing the applications of HPMA polymer in combination with diagnostic agents. For example, a passive targeted HPMA-doxorubicin (DOX) conjugate labeled with I-131 has been studied in Phase I clinical trial [37]. In another study, targeted HPMA drug-conjugate was prepared as theranostic nanomedicine. HPMA with Gadolinium was prepared by copolymerization method, attached with Arg-Gly-Asp (RGD) and labeled with In-111as diagnostic agent [38]. Most recently, Yuan et al. [39] synthesized poly(HPMA) based theranostic copolymers loaded with $\mathrm{Cu}-64$ (i.e., intrinsic theranostic agent) and RGD was used as targeting ligand for targeting tumor angiogenesis (Figure 3A). The tumor localization of the drug-polymer conjugate was evaluated in mice bearing human prostate cancer xenografts by positron emission tomography (PET). PET quantitative analysis demonstrated that tumor $\mathrm{Cu}-64$ radioactivity in tumor-bearing mice $3 \mathrm{~h}$ following i.v. injection of the drug-polymer conjugate was significantly higher than the tumor $\mathrm{Cu}-64$ radioactivity in tumor-bearing mice injected with the non-targeted drug-polymer conjugate. The drug-polymer conjugate showed 1 fold improvement in the pharmacokinetics of Cu-64 in tumor [39].

\subsection{Polymeric/magnetic nanoparticles}

Polymeric nanoparticles from biodegradable polymers have been extensively studied for various therapies $[9,40,41]$. The polymeric blocks were used to prolong the circulation half-life due to lower phagocytic uptake [42-44]. Polymeric nanoparticles are now used in theranostic applications for the targeted co-delivery of diagnostic and therapeutic agent owing to their biocompatibility, storage stability, protection of loaded drug/diagnostic agent and controlled/sustained release [43]. Biodegradable polymers are used for nanoparticles preparation to provide biological compatibility with less cytotoxicity. The biodegradable polymeric nanoparticles showed their advantage over micelles and liposomes by their excellent storage stability [42]. Poly(D,L-lactide-co-glycolide) (PLGA), PLA-TPGS and poly(D,L-lactide-co-glycolide) -polyethylene glycol (PLGA-PEG) are the mostly reported biodegradable polymers for the fabrication of theranostic polymeric nanoparticles [45,46]. PLA and PLGA will be easily hydrolyzed into their monomers (i.e., lactic acid or glycolic acid) and eliminated from human systems via metabolic pathways. The polymeric nanoparticles were prepared by either polymerization of monomers (e.g., emulsion method) or dispersion of polymers (e.g., nanoprecipitation, salting out, and solvent evaporation methods) [45-49]. 


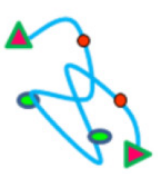

A

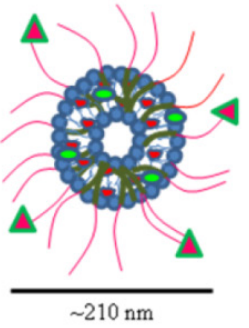

E

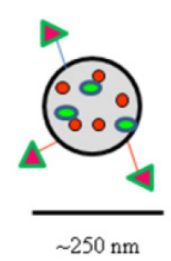

B

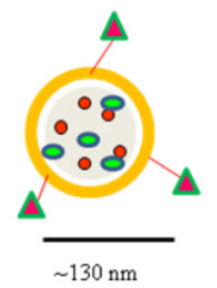

C

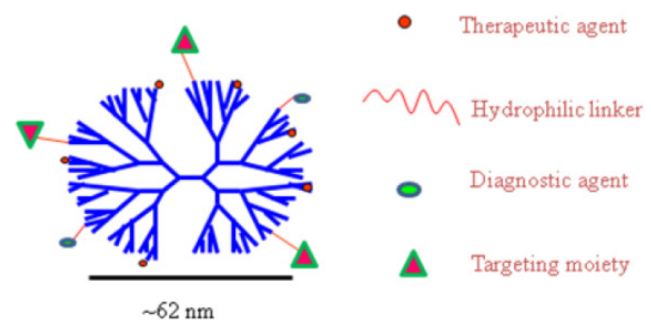

D

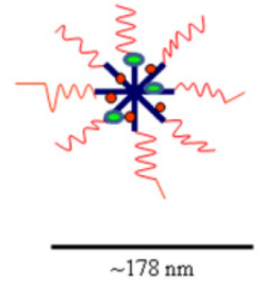

$\mathrm{F}$

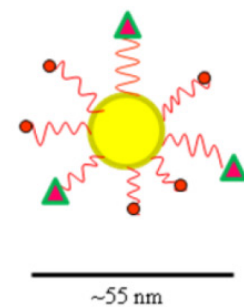

G

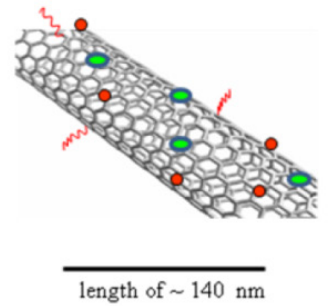

$\mathrm{H}$

Figure 3. Schematic diagram of theranostic A) polymer-drug conjugate; B) polymeric nanoparticle; C) solid lipid nanoparticle; D) dendrimer; E) liposome; F) micelle; $\mathrm{G}$ ) gold nanoparticle; and $\mathrm{H}$ ) carbon nanotube

The magnetic nanoparticles are iron oxide nanoparticles made from magnetite or hematite [50]. Iron oxide nanoparticles were highly used owing to their superparamagnetic effects and acceptable biocompatibility. Co-precipitation and thermal decomposition methods are the facile and convenient methods to synthesize iron oxide nanoparticles. They mainly act as theranostic in cancer treatment alone or loaded into other nanoplatforms like polymeric nanoparticles, liposomes etc [50-54]. In addition to intrinsic theranostic property, magnetic nanoparticles have prominent transverse relaxation time (T2) among diagnostic agents for MRI and play role in cancer therapy as hyperthermia agent and immunotherapeutic platform for autoimmune diseases [54,55]. The surface coating of iron oxide nanoparticles using dextran, PEG, oleic acid or surfactant improves the stability and water dispersibility of the nanoparticles $[56,57]$.

In one study, Zhang et al. [58] prepared cholic acid conjugated PLGA nanoparticles with co-administration of autophagy inhibitors such as 3-methyladenine and chloroquine. The IC50 values of the docetaxel formulated in PLGA nanoparticles in combination with $10 \mathrm{mM}$ 3-methyladenine or $30 \mathrm{mM}$ chloroquine are found 5.7 or 8.0 fold more effective for the nanoparticle drug delivery after $24 \mathrm{~h}$ treatment. The xenograft tumor volume of the severe combined immunodeficiency (SCID) mice after 20 day treatment with the PLGA nanoparticles formulation combined with 3-methyladenine or chloroquine are found to be only about a half in comparison with the PLGA nanoparticles formulation only [58]. Thus, the incorporation of autophagy inhibitor in future theranostic platform, can improve the co-delivery of diagnostic and therapeutic agent. In addition, they found a new mechanism of cancer cells to have polymeric nanoparticles captured and degraded by auto-lysosomes, which is thought to be autophagy pathway [58]. Previous studies reported that polymeric nanoparticles are internalized into the cells through endocytosis, and then can be transported into lysosomes for degradation only by endosome pathway (Figure 4) [13, 58].

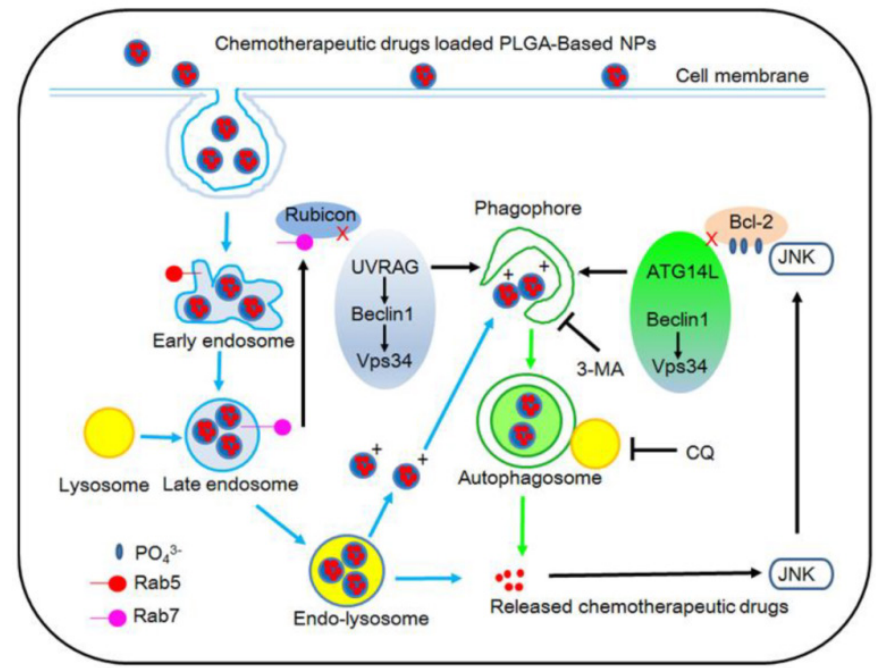

Figure 4. Schematic representation of the degradation pathway of PLGA-based nanoparticles in cancer cell. Reproduced with permission from Figure 8 of ref. [58] Elsevier Ltd, ( (2014). 
In our group, we demonstrated PLA-TPGS based theranostic platforms [59]. A good example of PLA-TPGS based theranostic is PLA-TPGS and acid functionalized D-a-tocopheryl polyethylene glycol 1000 succinate (TPGS-COOH) copolymer blend nanoparticles prepared with folate targeting (Figure 3B). The nanoparticles were loaded with both docetaxel and quantum dots. The targeting effect of quantum dots loaded PLA-TPGS/TPGS-COOH nanoparticles was studied in both MCF-7 breast cancer cells which overexpresses folate receptors and NIH-3T3 fibroblast cells which are expressing low quantity of folate receptors. The studies showed higher internalization/cytotoxicity of folate-decorated quantum dots loaded PLA-TPGS/TPGS-COOH nanoparticles by MCF-7 breast cancer cells than by the NIH 3T3 fibroblast cells [59]. Therefore, copolymers of TPGS conjugated with targeting ligands will be a promising theranostic tool for targeted diagnosis and therapy. Later, we developed a multimodal imaging system by co-encapsulating superparamagnetic iron oxide nanoparticles for MRI and quantum dots for fluorescence imaging [18]. The nanoparticles of PLA-TPGS were fabricated to combine their advantages and to promote a sustained and controlled imaging with passive targeting to the cancer cells. This novel strategy reduced the toxicity of the individual contrast agents and improved their biocompatibility and cellular uptake. The xenograft model was also conducted for biodistribution of the quantum dots and iron oxides loaded PLA-TPGS nanoparticle among the various organs, which showed greatly enhanced tumor imaging. The ex vivo fluorescent images analysis showed the significant percentage fluorescent intensity increase of $67.1 \%$ in liver, $51.5 \%$ in kidney and $152.8 \%$ in tumor. The surface adsorption of nanoparticles in blood-brain barrier showed more fluorescent signal for brain samples than other organs. Also, percentage fluorescent intensity increase in brain was observed less for treated group owing to poor improvement in biodistribution of the quantum dots and iron oxides loaded PLA-TPGS nanoparticle across the blood-brain barrier (Figure 5). The multimodal imaging system shows great advantages of both contrast agents making the resultant probe highly sensitive with good depth penetration for longer duration up to $6 \mathrm{~h}$, which confirms the diagnosis obtained from each individual imaging [18]. Further, therapeutics encapsulation and ligand conjugation with this multimodal system was suggested to develop an advanced multimodal theranostic nanomedicine. As a multimodal approach, Medarova et al. [60] developed iron oxide nanoparticles for simultaneous in vivo imaging and siRNA delivery into tumors by high resolution MRI and near infrared (NIR) in vivo optical imaging.
The siRNA was coupled with aminated dextran particles using $\mathrm{N}$-succinimidyl-3-(2-pyridyldithio) propionate as a bridge compound. Further, myristoylated polyarginine peptide, a membrane translocation peptide, and the NIR dye Cy5.5 were conjugated to iron oxide nanoparticles surface. By tracking these probes in vivo by MRI and NIR fluorescence optical imaging, the delivery of siRNA and its silencing efficiency was monitored for $48 \mathrm{~h}$ by dextran coated iron oxide nanoparticles (Figure 6) [60].

\subsection{Solid lipid nanoparticles}

Solid lipid nanoparticles are a safe and effective alternative colloidal matrix carrier to conventional emulsions, liposomes, and polymeric nanoparticles for intravascular delivery [9]. They are made up of solid hydrophobic core containing dissolved or dispersed drug. Solid lipid nanoparticles are nanomedicine made from biocompatible lipids (e.g., triglycerides) which are solid at room temperature. The commonly used methods of preparation are hot and cold homogenization. Solid lipid nanoparticles gain access to the blood compartment easily because of their small size and lipophilic surface. The size range of less than $100 \mathrm{~nm}$ allows solid lipid nanoparticles to cross tight-endothelial cells of blood-brain barrier for brain targeting. The high drug loading efficiency renders the drug stable in solid lipid matrix, and provides controlled release up to several weeks [61-63]. Similar to other nanomedicines, solid lipid nanoparticles are used for targeted co-delivery of diagnostic and therapeutic agent as theranostic platform [63, 64]. Recently, lymphatic delivery of solid lipid nanoparticles emerged as a technology to provide better transport into the lymphatics resulting in enhanced oral bioavailability of therapeutic agents [65]. For example, Shuhendler et al.[66] reported a novel targeted NIR light-emitting solid lipid nanoparticles by conjugating $\mathrm{a}(\mathrm{v}) \quad \beta(3)$ integrin-specific ligand i.e., cyclic Arg-Gly-Asp (cRGD) and loaded NIR quantum dots for live animal imaging [66]. They were injected intravenously to nude mice bearing xenograft breast tumors. Tumor micropharmacokinetics and whole animal biodistribution by optical imaging of the formulations were studied. The active tumor targeting with cRGD showed significantly greater distribution in the liver, spleen, and kidneys than the non-targeted solid lipid nanoparticles. The enhanced specificity and retention of RGD-solid lipid nanoparticles in tumor neovasculature for $120 \mathrm{~h}$ suggested the potential use of the solid lipid nanoparticles for theranostic application of neovascular-specific therapies and imaging.

In another work, Bae et al. [67] reported quantum dots loaded solid lipid nanoparticles for anti- 
cancer theranostics with synergistic/multimodal therapeutic effects of paclitaxel and siRNA (Figure 3C). The low density lipoprotein (LDL)-mimetic solid lipid nanoparticles were prepared with stable core/shell nanostructure incorporating quantum dots and paclitaxel within the lipid shell while anionic siRNA molecules are electrostatically complexed with the outer surface of solid lipid nanoparticles. The prepared solid lipid nanoparticles/siRNA complexes efficiently delivered both paclitaxel and Bcl-2 target-

A B ing siRNA into human lung carcinoma cells and exhibited synergistic anticancer activities by triggering caspase-mediated apoptosis. The strong fluorescence intensity of quantum dots within solid lipid nanoparticles enables in situ visualization and intracellular translocation of solid lipid nanoparticles in cancer cells. The experiment showed that LDL-mimetic solid lipid nanoparticles as a multifunctional/multimodal and optically traceable nanocarrier for efficient anticancer theranostics [67].
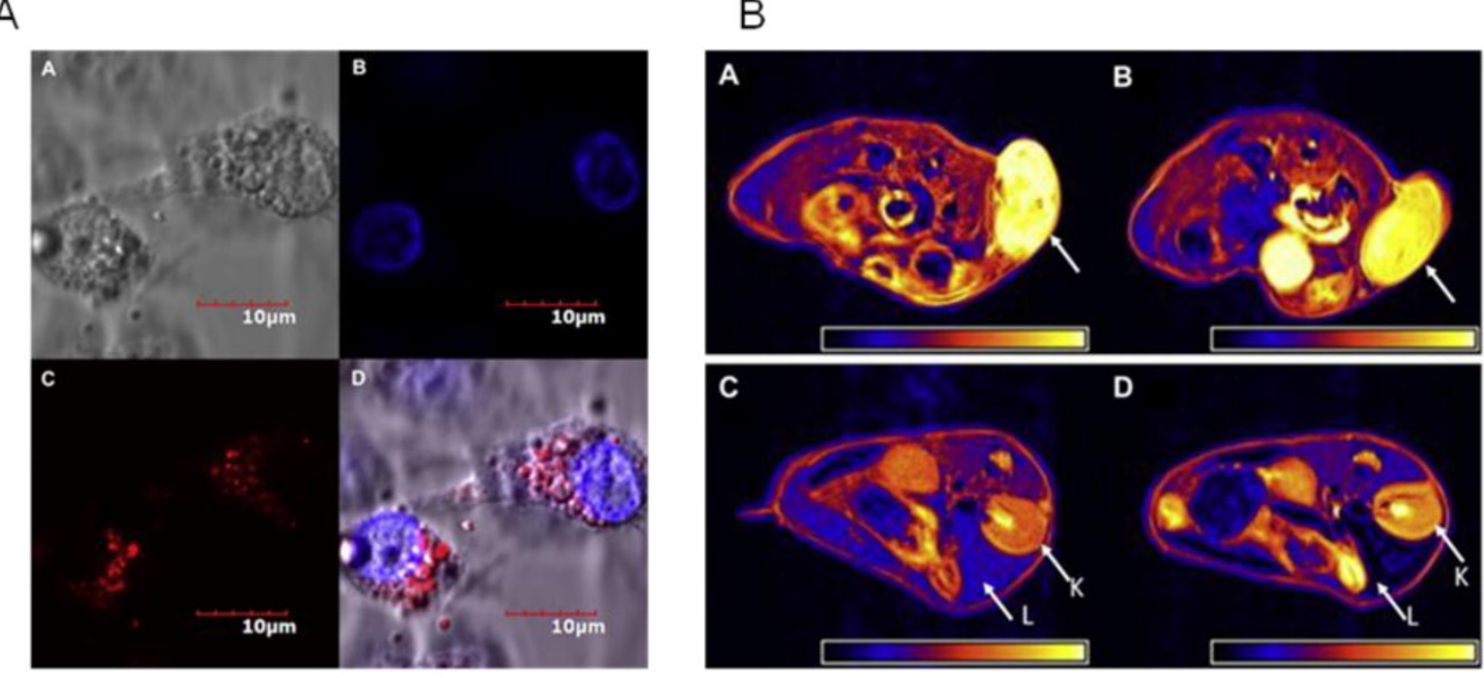

D

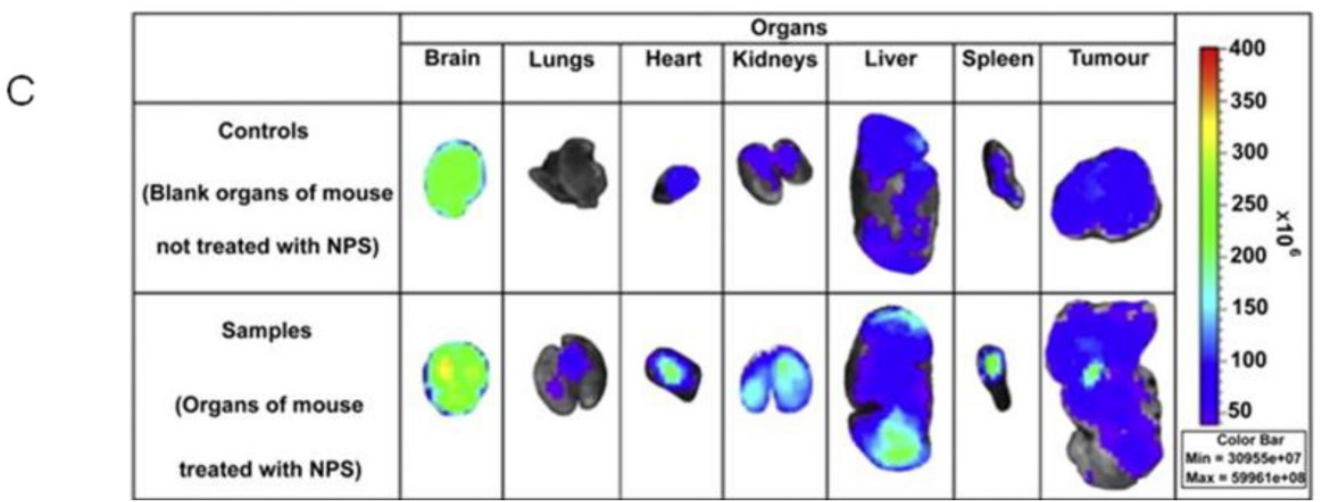

Figure 5. Multimodal imaging of polymeric nanoparticles A) Confocal images of MCF-7 cells treated with the quantum dots and iron oxides loaded PLA-TPGS nanoparticle in vitro (scale bar $=10 \mu \mathrm{m}$ ). A: Bright field image of cells. B: Blue coded stained nuclei. C: Red coded quantum dots from nanoparticles in cytoplasm. D: Complete overlapped image. B) Axial MRI image sections of the MCF-7 grafted tumor bearing mice. Images A and B show the part of the tumor (shown by the arrow) before and after $6 \mathrm{~h}$ of administration of the quantum dots and iron oxides-loaded PLA-TPGS nanoparticle into the mice. Images $\mathrm{C}$ and $\mathrm{D}$ show the kidney (K) and liver (L) part of the mice before and $6 \mathrm{~h}$ after the administration of the PLA-TPGS nanoparticle formulation of quantum dots and iron oxides (dosage: I.5 mg of Cd/kg of body weight or equivalent of $6.0 \mathrm{mg}$ of Fe/ $\mathrm{kg}$ body weight). The decrease in intensity in the regions of the tumor and liver can be noticed in comparison with the color scale aside. C) Fluorescent images of the various organs. Upper row: control. Lower row: Organs of the mouse treated with the quantum dots and iron oxides-loaded PLA-TPGS nanoparticle (dosage: $1.5 \mathrm{mg}$ of Cd/kg of body weight or equivalent of $6.0 \mathrm{mg}$ of Fe/kg body weight). Reproduced with permission from Figure 2, 5, 6 of ref. [18] Elsevier Ltd, (C) (201I). 
a

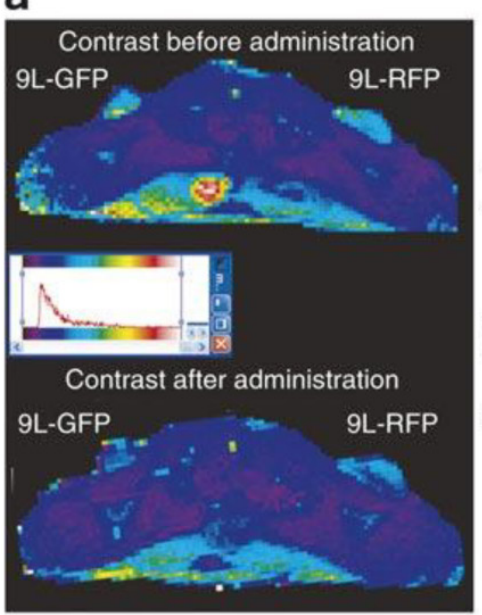

C

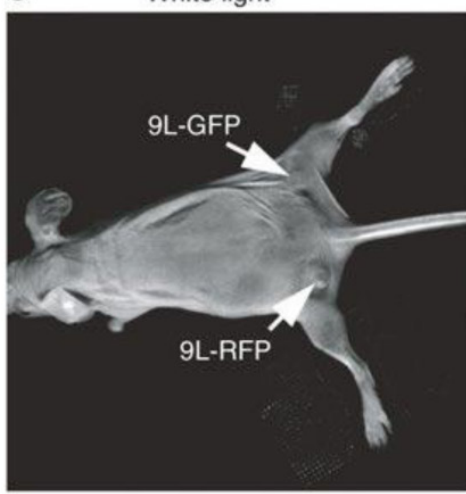

d

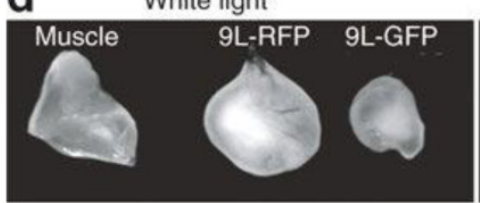

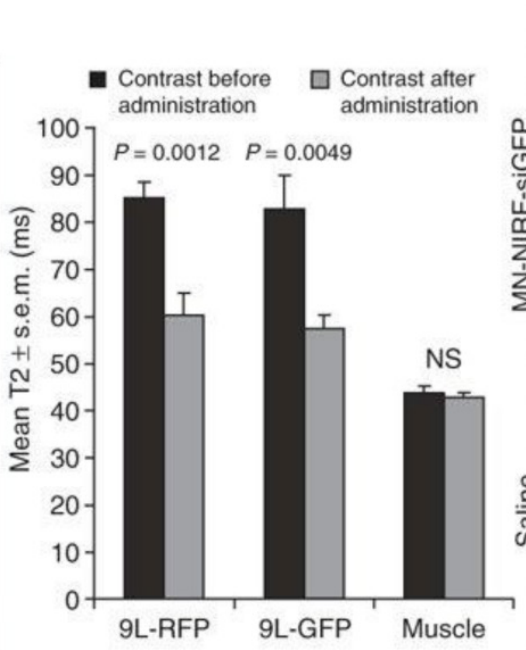

NIRF

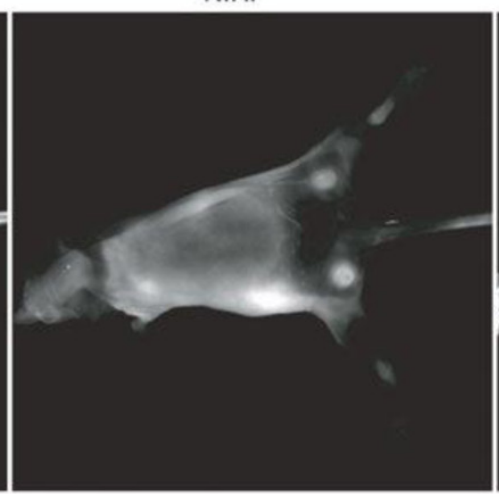

NIRF

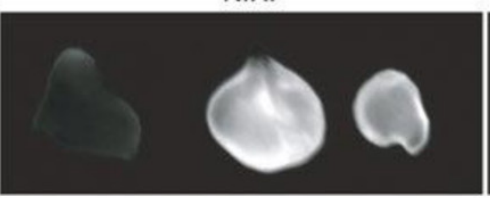

b
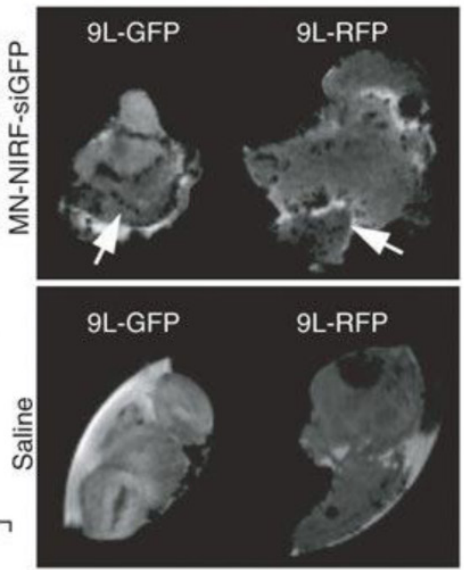

Color coded NIRF

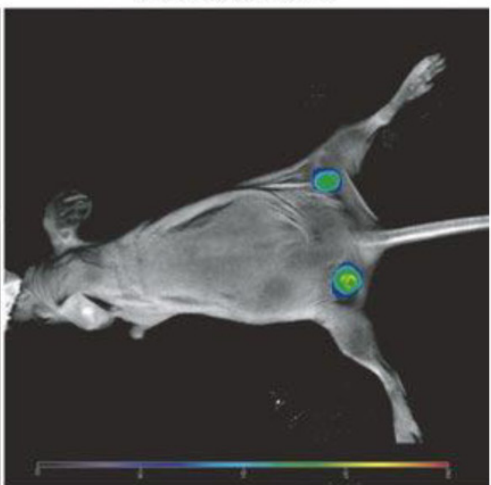

Color coded NIRF

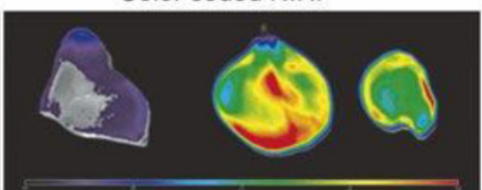

Figure 6. Theranostic approach using dextran coated iron oxide nanoparticles (a) In vivo MRI was performed on mice bearing bilateral green fluorescent protein (9L-GFP) and red fluorescent protein (9L-RFP) tumors before and $24 \mathrm{~h}$ after nanoparticles administration. After injection of the probe, there was a significant drop in T2 relaxivity associated with the tumors. Note that T2 relaxation times of muscle tissue remained unchanged. (b) Ex vivo high-resolution MRIs of excised tumors (78 $\mathrm{m}$ isotropic). Distinct foci of signal loss (arrows), reflecting probe accumulation, were easily identifiable in tumors derived from mice injected with the probe but not from saline-injected controls. (c) In vivo NIR optical imaging of the same mice as in a produced a high-intensity NIR signal associated with the tumors. This confirmed the delivery of the nanoparticles probe to these tissues. (d) Ex vivo NIR optical imaging demonstrated a significantly higher fluorescence in tumors than in muscle tissue $(P=0.0058)$. Reproduced with permission from Figure 3 of ref. [60], Macmilan Publishers Ltd: Nature Medicine, () (2007).

\subsection{Dendrimers}

Dendrimers are synthetic nanomedicine that comprises a highly branched spherical polymer. Dendrimers used in nanotheranostics are usually 10 to 100nm [68]. Dendrimers can be synthesized starting from the central core toward the periphery (divergent synthesis) or in a top-down approach starting from the periphery (convergent synthesis). They are synthesized by the repetitive addition of branching units to an amine core (ethylene diamine or ammonia). The basic structure of dendrimers is a repeated branching around the core resulting in an early-perfect three-dimensional (3D) geometrical pattern. Dendrimers showed toxicity by disrupting cell mem- branes due to their positive surface charge. In some cases, dendrimers encapsulated drug for targeted drug delivery tends to release rapidly, before they reach a targeted site $[69,70]$. By controlling the degree of polymerization, dendrimers can be synthesized in various sizes, molecular weights and chemical compositions [71, 72]. The higher generation dendrimers resemble spherical shape, having a number of cavities and branches to hold both therapeutic and diagnostic agents for theranostic application. The fifth generation dendrimers (G5) having more hydrophobicity are generally preferred owing to enhanced drug stability in dendrimers [69-72]. In one study, a replication-deficient adenovirus serotype 5 carrying the so- 
dium-iodide symporter (hNIS) gene was coated with poly(amidoamine) dendrimers generation 5 (PAMAM-G5) and investigated for transduction efficacy by I-123 scintigraphy in a liver cancer xenograft mouse model. After dendrimer coating, replication-deficient adenovirus serotype 5/hNIS demonstrated partial protection from neutralizing antibodies and enhanced transduction efficacy in coxsackie-adenovirus receptor-negative cells in vitro. In vivo I-123 scintigraphy of nude mice revealed significantly reduced hepatic transgene expression after i.v. injection of dendrimer-coated replication-deficient adenovirus serotype 5/hNIS [73]. Further, Saad et al. [74] designed, characterized, and evaluated a theranostic dendrimer for in vitro and in vivo. These nanocarriers delivered the paclitaxel and diagnostic agent (Cy5.5). A synthetic analog of luteinizing hormone-releasing hormone (LHRH) peptide targeted to receptors overexpressed on the membrane of cancer cells was attached to the dendrimer as a tumor targeting moiety. Significant differences were found between various studied non-targeted carriers in their cellular internalization, cytotoxicity, tumor and organ distribution and anticancer efficacy. High cellular uptake of tumor targeted theranostic dendrimer was accompanied by targeted accumulation of paclitaxel which reduced the adverse effect on healthy organs and increased the specificity and sensitivity of cancer imaging using fluorescent probes. It shows the theranostic nature of LHRH receptor targeted dendrimer [74].

In another study, Taratula et al. [75] developed a novel dendrimer-based theranostic platform for tumor-targeted delivery of phthalocyanines (Pc) (Figure $3 \mathrm{D})$. The preparation procedure involved the modification of the Pc molecule with a hydrophobic linker, which significantly enhances physical encapsulation of the hydrophobic drug into a generation 4 polypropylenimine (PPIG4) dendrimer. To improve biocompatibility and tumor-targeted delivery, the surface of the Pc-PPIG4 complexes was additionally modified with PEG and LHRH peptide, respectively. The synthesized phthalocyanine derivative entrapped in the dendrimer-based nanocarrier exhibits a distinct NIR absorption $(700 \mathrm{~nm})$ and fluorescence emission (710 and $815 \mathrm{~nm}$ ), required for an efficient photodynamic therapy and fluorescence imaging. It was demonstrated that subcellular localization in vitro and organ distribution in vivo of the developed nanocarrier can be determined based on the intrinsic fluorescence properties of encapsulated phthalocyanine. The prepared formulation showed high cytotoxicity and significant photodynamic therapy up to $24 \mathrm{~h}$. The in vitro and in vivo imaging experiments revealed that the LHRH targeted theranostic dendrimer is capable of an efficient internalization into cancer cells as well as tumor accumulation. The study revealed the significant potential of dendrimers as an efficient NIR theranostic agent [75]. Likewise, dendrimers have been under development for recent years and proven to be successful theranostic carriers in number of preclinical studies.

\subsection{Liposomes}

Liposomes are small bilayer vesicles enclosing aqueous compartment that can be produced using amphiphilic phospholipids and cholesterol [76]. Liposomes are spherical, vary in size most are $400 \mathrm{~nm}$ or less. Liposomes are effective platforms for drug/diagnostic delivery owing to their size, hydrophobic and hydrophilic character, biocompatibility, biodegradability, low toxicity and immunogenicity. Commonly, liposomes with smaller diameter (80-200 $\mathrm{nm})$ with neutral or negative charge are used for theranostic purpose [77-79]. The major three types of liposome preparation methods are mechanical dispersion, solvent dispersion and detergent removal. However, the potential drawbacks of liposomes are low drug loading efficiency, batch-to-batch variation in manufacturing, and poor stability [77-79]. The nanosized diagnostic agents such as iron oxide nanoparticles, quantum dots and gold nanoparticles can be entrapped within the theranostic liposomes and the therapeutic agent can be either encapsulated in the core or embedded in the lipophilic bilayer shell [80-85]. The advanced theranostic liposomes are conjugated with molecular biomarkers for targeting effect. To overcome opsonization by the immune system and fast elimination from blood circulation, stealth liposomes i.e., PEG-coated liposomes, were formulated with stability and longer half-life in blood $[84,86]$. Recently, our group prepared liposomes surface-coated with TPGS and loaded docetaxel and evaluated for possibility against brain tumor by in vitro cell line studies with PEGylated liposomes, conventional nude liposomes (without TPGS coating) and TPGS coated liposomes. The TPGS-coated liposomes showed higher efficacy than PEGylated liposomes [87]. Muthu et al. [88] prepared TPGS coated theranostic liposomes containing docetaxel and quantum dots with and without targeting moieties (Figure 3E). Folic acid was used as targeting probe to target folate receptor overexpressing MCF-7 breast cancer cell lines. The higher cellular uptake and cytotoxicity of targeted theranostic liposomes was observed in comparison to non-targeted liposomes (Figure 7). Along similar lines, Wen et al. [89] have developed quantum dots and apomorphine incorporated theranostic liposomes to eliminate uptake by the liver and to enhance brain targeting. The formulation was evaluated for in vitro brain endothelial cell uptake 
and in vivo bioimaging. In vitro cellular uptake study confirmed the delivery of liposomes through receptor mediated endocytosis routes. A significant distribution was observed for theranostic liposomes compared to free quantum dots. The brain uptake was increased 2.4 fold after the quantum dots were incorporated into liposomes. Quantum dots uptake was decreased in heart and liver by liposomes delivery. The fluorescence in the brain observed up to $1 \mathrm{~h}$ for the liposome treated group. However, free quantum dots was rapidly eliminated from the brain and retained more in liver up to $35 \mathrm{~min}$. The suggested mechanisms for the cellular uptake were clathrin-and caveola-mediated endocytosis [89]. In most recent work, Wen et al. [90] prepared theranostic liposomes loaded with quantum dots camptothecin and iri- notecan for simultaneous bioimaging and drug delivery. The different formulations, including cationic, PEGylated and deformable liposomes, were compared for their theranostic efficiency. The cytotoxicity and cell migration assay demonstrated superior activity of cationic liposomes compared with other carriers. Based on the results, cationic liposomes had higher efficiency to accumulate in solid tumors for exhibiting fluorescent signals compared to other controls. The cationic liposomes showed the brightest fluorescence in tumors than control liposomes. This signal could last for up to $24 \mathrm{~h}$ for cationic nanosystems. The versatile and tunable properties of the liposomes made them efficient theranostic delivery for extended duration.
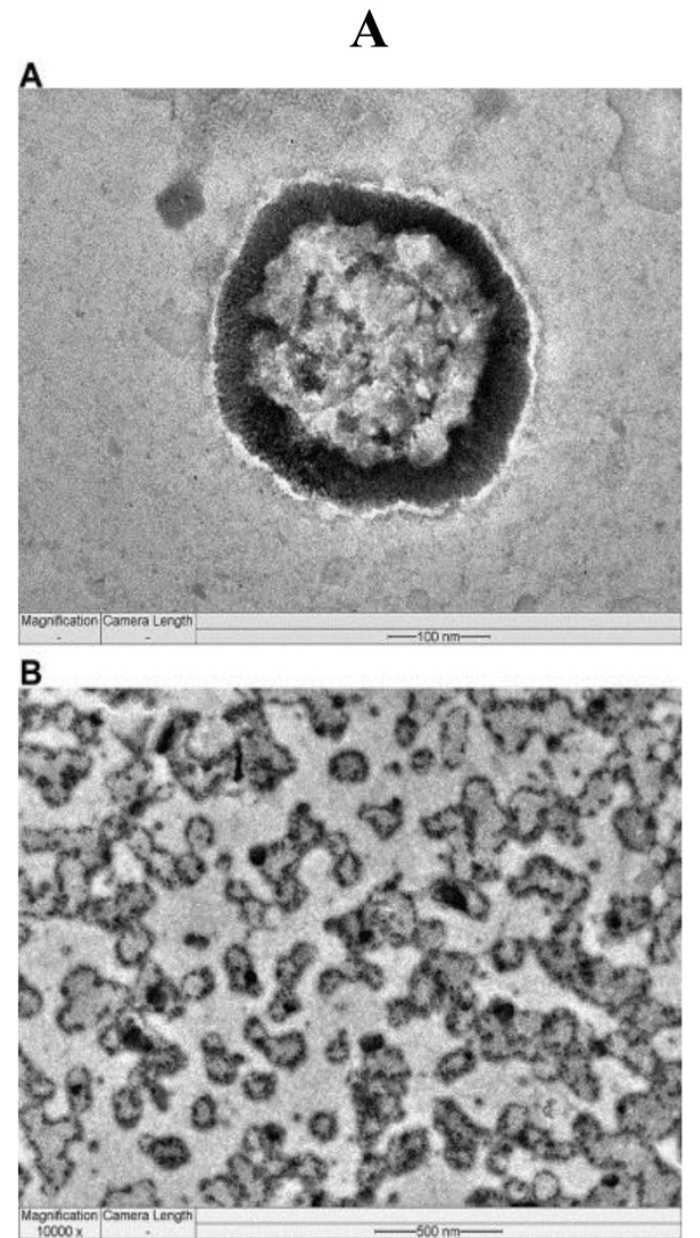

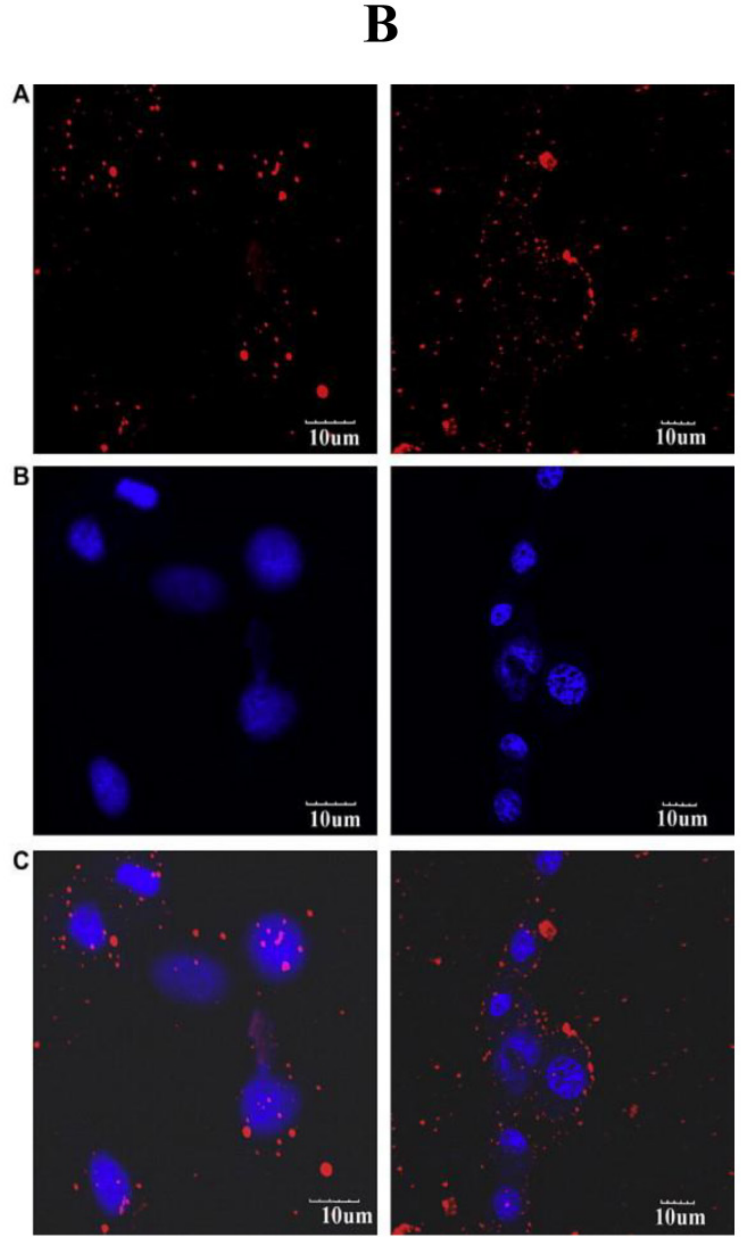

Figure 7. In vitro theranostic delivery using TPGS liposomes A) Field-emission transmission electron microscopy image of (A) an individual quantum dots loaded-TPGS coated liposome in $100 \mathrm{~nm}$ scale, and (B) multiple quantum dots-loaded TPGS coated liposomes after the storage in $500 \mathrm{~nm}$ scale. B) Confocal laser scanning microscopy images using MCF-7 cells after $2 \mathrm{~h}$ incubation with the non-targeted TPGS based multi-functional liposomes (left column) and targeted TPGS based multi-functional liposomes (right column). Row (A): Quantum dots showing the red fluorescence from liposomes distributed in cytoplasm, (B): Channels showing the blue fluorescence from dye stained nuclei, and (C): Merged channels of quantum dots and blue dye. Scale bar $=10 \mu \mathrm{m}$. Reproduced with permission from Figure 3, 6 of ref. [88] Elsevier Ltd, ( $\odot(2012)$. 


\subsection{Micelles}

Micelles are self-assembling colloidal structure with a hydrophobic core and hydrophilic shell. They have narrow size distribution and less than $100 \mathrm{~nm}$ in size [91]. The micelles are another safer alternative for parenteral administration of poorly water-soluble materials [92, 93]. The stability of the micelles depends on strong cohesive force between drug and polymer core as well as cross linking of the shell or core. They are generally prepared by direct dissolution method and organic solvent method [94]. Therapeutic/diagnostic agents can be loaded into hydrophobic core of micelles and the outer hydrophilic layer with targeting agent, which can then be administered intravenously [95-98]. In some cases, targeted drug delivery by micelles are hindered by premature drug release from the micellar nanomedicine before they reaches the molecular targets. The theranostic micelles of particle size less than $50 \mathrm{~nm}$ in diameter avoid renal exclusion, reticulo endothelial system and provide them with enhanced endothelial cell permeability in solid tumors [99, 100]. Micelles are efficient platform for cancer drug delivery e.g., Genexol-PM ${ }^{\mathrm{TM}}$ which is a paclitaxel loaded micellar formulation by using poly(ethylene glycol)-block-poly(D,L-lactide) [101]. Some clinical trials were already performed that evaluated the safety and efficacy of Genexol-PM ${ }^{\mathrm{TM}}$ [102-104].

TPGS is an alternative material for the preparation of drug/diagnostic material loaded targeted/non-targeted micelles [105-107]. We prepared theranostic TPGS micelles containing superparamagnetic iron oxides for diagnosis and therapy of cancer (Figure 3F). It had improved thermal and magnetic properties, in-vitro cellular uptake, cytotoxicity and in-vivo imaging effects in comparison to commercial Resovist ${ }^{\circledR}$ and Pluronic F127 micelles. The prepared TPGS micelles were found to be highly monodisperse, suitable size range and stable. The cellular uptake and cytotoxicity were investigated in vitro with a MCF-7 breast cancer cell line for $24 \mathrm{~h}$. Cell viability was decreased after hyperthermia treatment using micelles (Figure 8). Also, T2-mapped images of xenograft grown on SCID mice showed that iron oxide loaded TPGS micelle had $\sim 1.7$ times and $\sim 1.05$ times T2 decrease at the tumor site compared to Resovist and Pluronic F127 micelle formulation, respectively (Figure 9) [108]. In another work, Kim et al prepared hyaluronic acid-DOX conjugate by the amide bond formation between amine groups of DOX and carboxylic groups of hyaluronic acid [109]. Hyaluronic acid-DOX conjugates self-assembled to micelle-like nanoparticles. The cytotoxicity study on cancer cells confirmed the anticancer effect of hyaluronic acid-DOX conjugate micelles. Further, micelles were coated with a gold half-shell by the thermal vapor deposition method, which might contribute to prolonging the residence time using gold surface. The gold half-shell-coated hyaluronic acid-DOX conjugate micelles are successful platform, may be exploited for future cancer theranostic applications [109]. Owing to stability, ease of preparation, hydrophobic encapsulation and success in preclinical and clinical studies, micelles are emerging platforms as theranostic delivery systems.

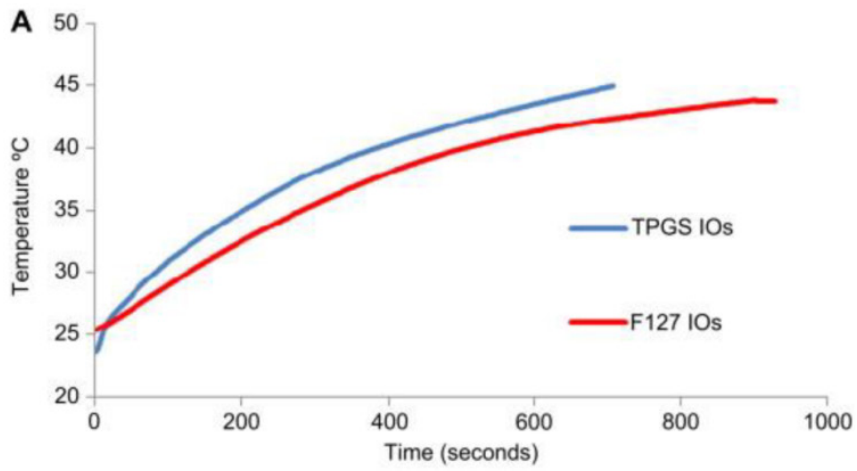

B
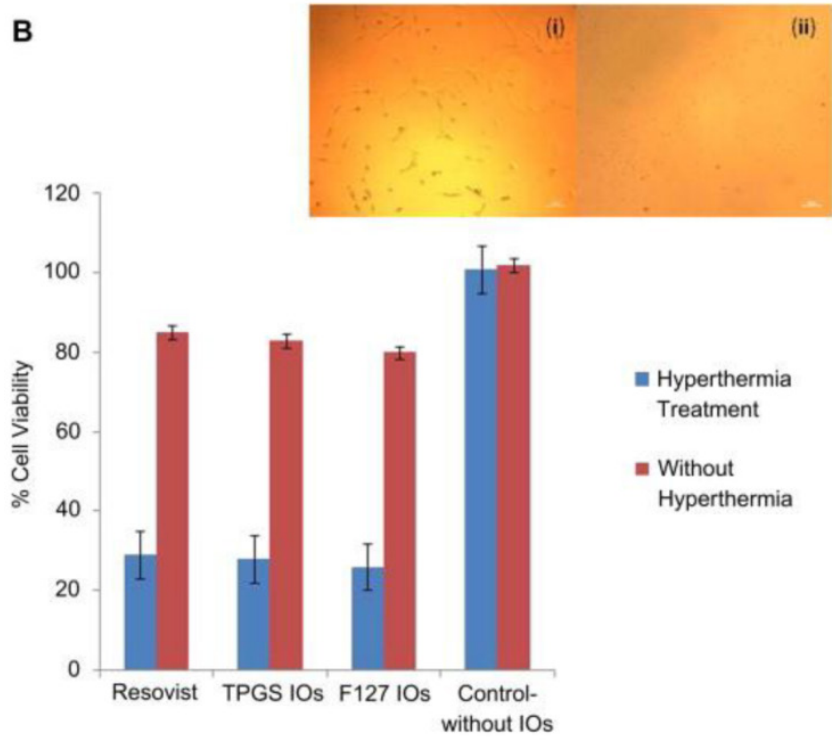

Figure 8. In vitro effect of theranostic micelles loaded with iron oxide nanoparticles (A) Hyperthermia study shows that the time-dependent temperature rise of I mg/ml of the iron oxide nanoparticles (IOs)-loaded TPGS and FI27 micelles on exposure to $89 \mathrm{kA} / \mathrm{m}$ alternating current field at $240 \mathrm{kHz}$ frequency. The specific absorption rate value for the IOs-loaded TPGS and FI27 micelles was found to be 51.4 and $25.5 \mathrm{Watt} / \mathrm{g}$. (B) The cytotoxic assay performed after hyperthermia treatment of MCF-7 cancer cells incubated with the IOs-loaded TPGS and FI27 micelle and Resovist ${ }^{\circledR}$. Hyperthermia treatment in all cases leads to significant cell death. Inset image (i) shows the cells incubated with the IOs-loaded TPGS micelles but no AC field applied and inset image (ii) shows the cells incubated with the IOs-loaded TPGS micelles and hyperthermia treatment using AC field. From (ii), it can be seen that after hyperthermia treatment, the cell loses viability and do not attach. Reproduced with permission from Figure 6 of ref. [108] Elsevier Ltd, (C) (20I I). 


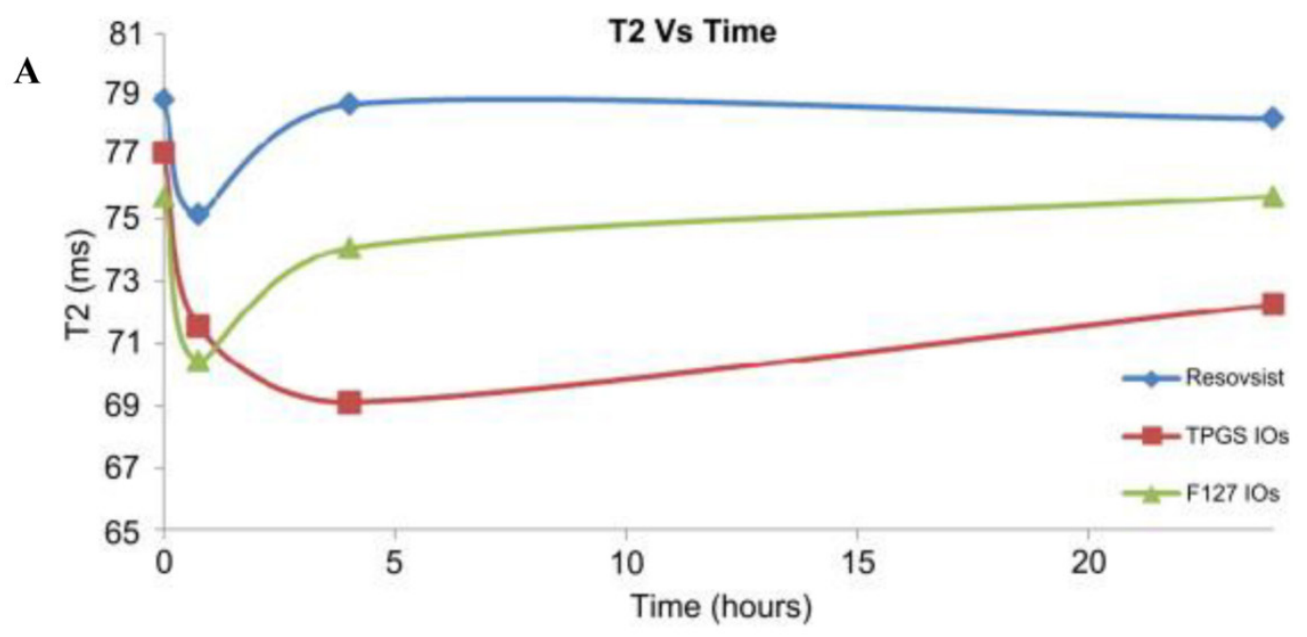

B

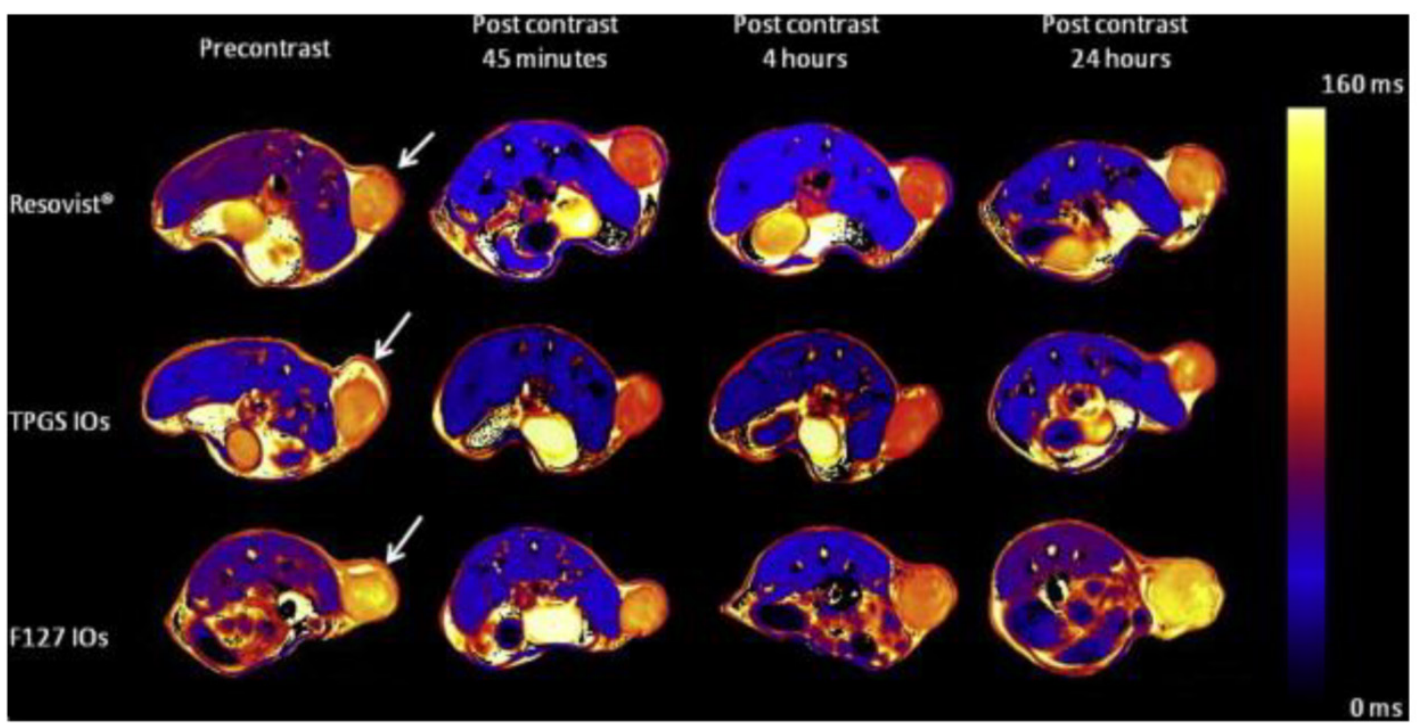

Figure 9. In vivo imaging of theranostic micelles loaded with iron oxide nanoparticles A) Graph showing the change of T2 values measured from MCF-7 xenograft tumor at the time point before and $45 \mathrm{~min}, 4 \mathrm{~h}$ and $24 \mathrm{~h}$ after injection of the iron oxide nanoparticles (IOs)-loaded TPGS and FI27 micelle and Resovist ${ }^{\circledR}$, respectively at a concentration of $5 \mathrm{mg}$ of $\mathrm{Fe} / \mathrm{Kg}$ of animal weight. It can be found that the $\mathrm{T} 2$ value decreases due to accumulation of $\mathrm{IOs}$ at tumor by $\sim 4.3 \%, 7.3 \%$ and $6.9 \%$ for Resovist ${ }^{\circledR}$, the IOs-loaded TPGS and FI 27 micelles respectively at 45 min after injection. B) T2 mapped images showing the MCF-7 xenograft tumor at the designated time point before and $45 \mathrm{~min}, 4 \mathrm{~h}$ and $24 \mathrm{~h}$ after injection of Resovists ${ }^{\circledR}$, the IOs-loaded TPGS and FI27 micelles respectively at a concentration of 5 mg of $\mathrm{Fe} / \mathrm{Kg}$ of animal weight. The change in T2 in the tumor (marked by the arrow) can be noticed in comparison with the T2 color scale aside. Reprinted with permission from Figure 9, 10 of ref. [108] Elsevier Ltd, (c) (201 I).

\subsection{Gold nanoparticles}

Gold nanoparticles based on gold cores are another versatile platform that provides desirable values for theranostic systems [110-113]. They are prepared with core size from 1.5 to $10 \mathrm{~nm}$, providing large area for efficient drug and ligand conjugations [114]. Gold nanoparticles are commonly synthesized by chemical treatment of hydrogen tetrachloroaurate. Gold nanoparticles can be conjugated with drug and targeting ligand as advanced theranostics that specifically recognizes the target receptor for active targeting [115, 116]. The therapeutic loading are achieved by either non covalent interaction (e.g. via electrostatic interaction) or covalent chemical conjugation of (i.e., organic
drug).The inherent features of gold nanoparticles include diagnostic property, tunable core size, monodispersity, low toxicity, large surface to volume ratio, surface plasmon absorption, ability to bind to biomolecules via $\mathrm{Au}-\mathrm{S}$ bonds, light-scattering properties and ease of fabrication [115-117]. Recently, gold nanoparticles showed the possibility of treating MDR tumors by targeted photothermal treatment in combination with a chemotherapeutic agent [118]. Heo et al. [119] described gold nanoparticles surface-functionalized with PEG, biotin, paclitaxel and rhodamine B linked beta-cyclodextrin (beta-CD)as theranostic platform. Paclitaxel formed inclusion complex with beta-CD which was then conjugated with gold nanoparticles. In vitro studies suggest that 
gold nanoparticles have higher affinity towards cancer cells such as HeLa, A549 and MG63 in comparison to NIH3T3 cells. Furthermore, gold nanoparticles displayed significant cytotoxic effect against HeLa cancer cells. The preliminary work indicates that the gold nanoparticles are feasible for use as theranostic nanomedicine.

In another work, smart theranostic gold nanoparticles were developed i.e., DOX was conjugated to gold nanoparticles via Au-S bond by using a peptide substrate, Cys-Pro-Leu-Gly-Leu-Ala-Gly-Gly (CPLGLAGG), which is specifically cleaved by the protease (Figure 3G). The animal studies showed that after injection of the functionalized gold nanoparticles to the tumor-bearing mice, the over-expressed protease in tumor tissue and intracellular glutathione have led to the rapid release of DOX from the functionalized gold nanoparticles. Here, enhanced efficacy on tumor growth inhibition and fluorescent imaging was realized simultaneously. The gold nanoparticles with stimulus responsive drug release property showed a promising smart theranostic application for concurrent cancer diagnosis and therapy [120].

\subsection{Carbon nanomaterials}

The carbon nanomaterials or nano-carbons have been studied for theranostic purpose owing to their unique physical and chemical properties [121]. Nano-carbons are classified into $\mathrm{sp}^{2}$-carbon nanomaterials such as zero dimensional (0D) fullerene, one dimensional (1D) carbon nanotubes (CNTs), two dimensional (2D) graphene and carbon dots (i.e., nano-clusters having size $<10 \mathrm{~nm})[121,122]$.The large surface area, ability to encapsulate therapeutic/diagnostic agent and suitability for surface modifications of CNTs made them suitable for theranostic applications [123-125]. The CNTs have cylindrical structure, based on their number of layer of graphene sheets CNTs are classified into single-walled carbon nanotubes (SWCNTs) or multi-walled carbon nanotubes (MWCNTs). CNTs are commonly synthesized by the discharge method, laser method, ball milling method and chemical vapor deposition method [125-130]. Recent years various synthetic approaches of CNTs functionalization have brought multifunctional-CNTs based platforms for theranostic applications. Robinson et al. [131] demonstrated an intrinsic theranostic application of intravenously injected SWCNTs ( average length $\sim 140 \mathrm{~nm}$ ) as photo luminescent agents for in vivo tumor imaging in the 1.0-1.4 $\mu \mathrm{m}$ emission region and as NIR absorbers and heaters at $808 \mathrm{~nm}$ for photothermal tumor elimination at lower doses (Figure 3H). An ex vivo resonance Raman imaging revealed the SWCNT distribution within tumors at a high spatial resolution. Fluorescence im- aging of SWCNTs shows confirmation of SWCNTs uptake by the tumors. The $100 \%$ tumor destruction was achieved for large numbers of photothermally treated mice without any toxic side effects after 6 months post-treatment. Highly effective tumor elimination with SWCNTs was achieved at 10 times lower injected doses and lower irradiation powers than other control preparation. These studies suggest that significant benefits of utilizing the intrinsic properties of biocompatible SWCNTs for theranostic applications [131].

In another study Das et al. [132] designed a theranostic MWCNTs by including acid-oxidised MWCNTs with four varieties of functional moieties: a fluorochrome (Alexa-fluor, AF488/647), targeting agent (i.e, folic acid), radionucleide (Technitium-99m), and methotrexate. The cellular uptake studies showed the selective internalization of theranostic MWCNTs by folate receptor positive human lung (A549) and breast (MCF 7) cancer cells through folate receptor mediated endocytosis. The tumor specific accumulation of targeted theranostic MWCNTs in xenografted mice after $24 \mathrm{~h}$ was 19.14 and 8.62 times higher in comparison to free and non-targeted theranostic MWCNTs. This study shows the controlled delivery of methotrexate from theranostic MWCNTs, receptor mediated delivery, optical detectability of fluorochromes, and radio-traceability of ${ }^{99 \mathrm{~m} T c}$. In summary, MWCNTs could be a promising multimodal theranostic platform for cancer diagnosis and therapy. In one study, graphene oxide (GO)-based theranostic nanohybrid is designed for tumor diagnosis and therapy [133]. The DOX is chemically conjugated to the poly(ethylenimine)-co-poly(ethylene glycol) (PEI-PEG) grafted GO via a matrix metalloproteinase-2 (MMP2)-cleavable peptide linkage. The intrinsic fluorescence property of DOX was quenched by GO under normal condition. During MMP2 enzyme over-expression in tumor, peptide was cleaved and released DOX for tumor therapy and concurrent fluorescence signal of DOX was used for in situ tumor cell imaging. Here, positively charged theranostic GO showed an efficient transfection for nanotherapeutics. This study opens a window for tumor treatment by theranostic approach with a combination of diagnosis and pathology responsive therapy [133]. Here, CNTs have demonstrated the efficacy during therapeutic and diagnostic delivery in preclinical level for future use.

\section{Conclusions and perspectives}

In this review, the recent developments of the various theranostic nanomedicines and their pre-clinical success were discussed. We noticed that in most cases, the interesting results of theranostic na- 
nomedicines in the literature are available only for in vitro studies. There are more challenges to be faced for their in vivo applications to pre-clinical and clinical level. A search on PubMed ${ }^{\circledR}$ shows that around 645 articles have been published in last 5 years within this research interest. However, almost all of them are focused on preparation, physico-chemical characterization and in vitro cell culture work of nanotheranostics with little in vivo evaluation. The in vivo data available are either therapeutic or diagnostic but not in combination of both. In fact, it is until recently that only a handful of investigations have revealed some in vivo facts of real theranostic nanomedicine which are summarized in Table 2 . The advanced nanotheranostic thus becomes an approach of future generation; with the variety of features in nanomedicine includes stimulus-responsive drug release (e.g., $\mathrm{pH}$, temperature, magnetism, and ultrasound), synergetic performance (e.g., siRNA delivery, combination therapy), multi-modal approach and quality performances (e.g., oral delivery, autophagy inhibition etc). Further, research efforts are needed to explore these concepts on available clinical platform of nanomedicines, towards the development of versatile and smart theranostic applications [134-136]. Quantum dots, gold nanoparticles and iron oxide nanoparticles loaded theranostic nanomedicines could be potential carrier to address some of these issues in near future. The iron oxide nanoparticles show excellent biosafety as they degrade and metabolize into the serum Fe pool to form hemoglobin or enter into metabolic pathway [137]. However, use of heavy metal based quantum dots as theranostic probes for human use is a major concern at present. It is a challenge to study the in vivo biodistribution and toxicology of quantum dots in animal systems that could potentially mimic its toxicity in human use. In future, fabrication of biocompatible, non-immunogenic, ultra-small size $(<5.0 \mathrm{~nm})$ quantum dots for nanomedicine loading that would be excreted through a renal clearance mechanism is highly awaited [21, 138, 139]. Carbon nanotubes exert potential to cause toxicities in vivo and in vivo. They produce oxidative stress (free radical formation) by causing lipid peroxidation which leads to inflammation and cell damage in major organs such as lungs and liver [140-144]. Also, oxidative stress induced cytotoxicity was reported for gold nanoparticles [145] but this cytotoxicity appears to be a common effect amongst other nanoparticles [146-151]. Therefore, theranostic carbon nanotubes and gold nanoparticles have raised a concern of safety for clinical use. Clearly, there are many options available for a nanotheranostic approach towards killing cancer cells but other non-cytotoxic nanoparticulate effects like induction of endothelial leakiness and crippling of cell migration during wound healing should not be neglected [152, 153]. Finally, multimodal theranostic nanomedicines (i.e., combination of multi diagnostics and drugs) could be advantageous for theranostic purposes. Theranostic approach using nanomedicine (i.e., nanotheranostics) which target molecular biomarkers with advanced designs is likely to contribute to increased precision for cancer therapy.

Table 2. In vivo efficacy analysis of theranostic nanomedicines.

\begin{tabular}{|c|c|c|c|c|c|c|}
\hline $\begin{array}{l}\text { Type of theranostic } \\
\text { nanomedicine }\end{array}$ & $\begin{array}{l}\text { Targeting/Therapeutic/ } \\
\text { Diagnostic agent }\end{array}$ & $\begin{array}{l}\text { Site of } \\
\text { administration }\end{array}$ & $\begin{array}{l}\text { Duration of } \\
\text { diagnosis }\end{array}$ & $\begin{array}{l}\text { Duration of } \\
\text { therapy }\end{array}$ & Fold of efficacy enhancement & $\begin{array}{l}\text { Refer- } \\
\text { ence }\end{array}$ \\
\hline $\begin{array}{l}\text { Drug-polymer } \\
\text { conjugates }\end{array}$ & $\mathrm{RGD} / 64 \mathrm{Cu}$ & i.v. & $3 \mathrm{~h}$ & $3 \mathrm{~h}$ & $\sim 1$ fold in tumor & [39] \\
\hline $\begin{array}{l}\text { Polymeric } \\
\text { nanoparticles }\end{array}$ & $\begin{array}{l}\text { Passive/Quantum dots/Iron oxide nano- } \\
\text { particles }\end{array}$ & i.v. & $6 \mathrm{~h}$ & -- & $\sim 1.5$ folds in tumor & [18] \\
\hline $\begin{array}{l}\text { Magnetic } \\
\text { nanoparticles }\end{array}$ & $\begin{array}{l}\text { Cy } 5.5 / \text { siRNA/ Iron oxide } \\
\text { nanoparticles }\end{array}$ & i.v. & $48 \mathrm{~h}$ & $48 \mathrm{~h}$ & In vivo silencing in tumor was observed & {$[60]$} \\
\hline $\begin{array}{l}\text { Solid lipid } \\
\text { nanoparticles }\end{array}$ & RGD/Lead selenide & i.v. & $120 \mathrm{~h}$ & -- & $\sim 1$ fold in tumor vasculature & {$[66]$} \\
\hline Dendrimers & LHRH/ Phthalocyanines & i.v. & $10 \mathrm{~h}$ & $24 \mathrm{~h}$ & $\begin{array}{l}\text { Significant fluorescent was observed in } \\
\text { tumor with cytotoxicity }\end{array}$ & {$[75]$} \\
\hline Liposomes & $\begin{array}{l}\text { Passive/Camptothecin/Irinotecan/ } \\
\text { Cholesterol }\end{array}$ & Intratumoral & $24 \mathrm{~h}$ & $24 \mathrm{~h}$ & $\begin{array}{l}\sim 6 \text { folds longer duration of detec- } \\
\text { tion/more drug concentration at tumor } \\
\text { site }\end{array}$ & [90] \\
\hline Micelles & Passive/Iron oxide nanoparticles & i.v. & $24 \mathrm{~h}$ & $24 \mathrm{~h}$ & $\begin{array}{l}\sim 1 \text { fold T2 value decreases with signifi- } \\
\text { cant cell death }\end{array}$ & [108] \\
\hline $\begin{array}{l}\text { Carbon } \\
\text { nanomaterials }\end{array}$ & $\begin{array}{l}\text { Folic acid/MWCNTs/ } \\
\text { Technitium-99m }\end{array}$ & i.v. & $24 \mathrm{~h}$ & $\begin{array}{l}1-15 \text { days } \\
\text { ( } 2 \text { doses per } \\
\text { week) }\end{array}$ & $\begin{array}{l}\sim 8.5 \text { folds higher than non- targeted } \\
\text { MWCNTs after } 24 \text { h of diagnosis with at } \\
\text { least } \sim 2 \text { folds higher tumor inhibition }\end{array}$ & {$[132]$} \\
\hline
\end{tabular}




\section{Abbreviations}

MDR, multi drug resistance; MRI, magnetic resonance imaging; PEG, polyethylene glycol; TPGS, D-a-tocopheryl polyethylene glycol 1000 succinate; HPMA, N- (2-hydroxypropyl) methacrylamide; DOX, doxorubicin; RGD, Arg-Gly-Asp; PET, positron emission tomography; PLGA, Poly (D,L-lactide-co-gl ycolide); PLA-TPGS, poly-lactic acid-D-a-tocopheryl polyethylene glycol 1000 succinate; PLGA-PEG, poly( D,L-lactide-co-glycolide)-polyethylene glycol; SCID, severe combined immunodeficiency; $\mathrm{T} 2$, transverse relaxation time; TPGS-COOH, acid functionalized D-a-tocopheryl polyethylene glycol 1000 succinate; cRGD, cyclic Arg-Gly-Asp; NIR, near infrared; LDL, low density lipoprotein; 3D, three-dimensional; hNIS, sodium-iodide symporter; PAMAM-G5, poly(amidoamine) dendrimers generation 5; LHRH, luteinizing hormone-releasing hormone; Pc, phthalocyanines; PPIG4, generation 4 polypropylenimine; beta- $\mathrm{CD}$, beta-cyclodextrin; CPLGLAGG, Cys-Pro-Leu-Gly-Leu-Ala-Gly-Gly; CNTs, carbon nanotubes; SWCNTs, single-walled carbon nanotubes; MWCNTs, multi-walled carbon nanotubes; GO, graphene oxide; IOs, iron oxide nanoparticles; NPs, nanoparticles; AIDS, acquired immunodeficiency syndrome; i.v., intravenous; 0D, zero dimensional; 1D, one dimensional; 2D, two dimensional; PEI-PEG, poly(ethylenimine)-co-poly(et hylene glycol); MMP2, matrix metalloproteinase- 2 .

\section{Acknowledgement}

This work is supported by the Singapore China Cooperative Research Project between the Agency of Science, Technology and Research (A*STAR), Singapore and Chinese Ministry of Science and Technology (MOST) (R-398-000-077-305) and the NUS FSF grant R-397-000-136-731 and FRC grant R-397-000-136-112. (PI: Feng SS, Leong DT). MS Muthu acknowledges the Department of Biotechnology (DBT), New Delhi, India, for the DBT-CREST Award (BT/IN/DBT-CREST Awards/26/MSM/2012-2013) to support his postdoctoral research in the Department of Chemical \& Biomolecular Engineering, National University of Singapore. L Mei acknowledges financial support from the National Natural Science Foundation of China (No. 31270019), Program for New Century Excellent Talents in University (NCET-11-0275), Natural Science Foundation of Guangdong Province (No. S2012010010046, S2012040006820), and Science, Technology \& Innovation Commission of Shenzhen Municipality (No. JCYJ20120616213729920, JCYJ20120614191936420, KQC201105310021A, JC201005270308A).

\section{Competing Interests}

The authors have declared that no competing interest exists.

\section{References}

1. Sumer B, Gao J. Theranostic nanomedicine for cancer. Nanomedicine (Lond). 2008; 3(2): 137-140

2. Deveza L, Choi J, Yang F. Therapeutic angiogenesis for treating cardiovascular diseases. Theranostics. 2012; 2(8):801-814.

3. Janib SM, Moses AS, MacKay JA. Imaging and drug delivery using theranostic nanoparticles. Adv Drug Deliv Rev. 2010; 62(11): 1052-1063.

4. Yu MK, Park J, Jon S. Targeting strategies for multifunctional nanoparticles in cancer imaging and therapy. Theranostics. 2012; 2(1):3-44

5. Xie J, Lee S, Chen X. Nanoparticle-based theranostic agents. Adv Drug Deliv Rev. 2010; 62(11):1064-79.

6. Ye Y, Chen X. Integrin targeting for tumor optical imaging. Theranostics. 2011; 1:102-126.

7. Feng SS. New-concept chemotherapy by nanoparticles of biodegradable polymers: where are we now? Nanomedicine (Lond). 2006 Oct;1(3):297-309.

8. Muthu MS, Feng SS. Theranostic liposomes for cancer diagnosis and treatment: current development and pre-clinical success. Expert Opin Drug Deliv. 2013; 10(2):151-155.

9. Muthu MS, Singh S. Targeted nanomedicines: effective treatment modalities for cancer, AIDS and brain disorders. Nanomedicine (Lond). 2009; 4(1):105-118

10. Muthu MS, Rajesh CV, Mishra A, et al. Stimulus responsive targeted nanomicelles for effective cancer therapy. Nanomedicine (Lond). 2009; 4(6): 657-667.

11. Zhao J, Mi Y, Feng SS. siRNA based nanomedicine. Nanomedicine (Lond). 2013; 8(6): 859-862.

12. Mei L, Zhang Z, Zhao L, et al. Pharmaceutical nanotechnology for oral delivery of anticancer drugs. Adv Drug Del Rev. 2013; 65(6): 880-890.

13. Smith BA, Smith BD. Biomarkers and molecular probes for cell death imaging and targeted therapeutics. Bioconjug Chem. 2012; 23(10): 1989-2006.

14. Lammers $\mathrm{T}$, Aime $\mathrm{S}$, Hennink WE, et al. Theranostic nanomedicine. Acc Chem Res. 2011; 44(10): 1029-1038.

15. Caldorera-Moore ME, Liechty WB, Peppas NA. Responsive theranostic systems: integration of diagnostic imaging agents and responsive controlled release drug delivery carriers. Acc Chem Res. 2011; 44(10): 1061-1070.

16. $\mathrm{Xu} \mathrm{C}$, Zhao $\mathrm{W}$. Nanoparticle-based monitoring of stem cell therapy. Theranostics. 2013; 3(8):616-617.

17. Ma $X$, Zhao $Y$, Liang XJ. Theranostic nanoparticles engineered for clinic and pharmaceutics. Acc Chem Res. 2011; 44(10): 1114-1122.

18. Tan YF, Chandrasekharan P, Maity D, et al. Multimodal tumor imaging by iron oxides and quantum dots formulated in poly (lactic acid)-D-alpha-tocopheryl polyethylene glycol 1000 succinate nanoparticles. Biomaterials. 2011; 32(11): 2969-2978.

19. Zhao L, Feng SS. Enhanced oral bioavailability of paclitaxel formulated in Vitamin E-TPGS emulsified nanoparticles of biodegradable polymers: in vitro and in vivo studies. J Pharm Sci. 2010; 99(8): 3552-3560.

20. Win KY, Feng SS. In vitro and in vivo studies on vitamin E TPGS-emulsified poly(D, L-lactic-co-glycolic acid) nanoparticles for paclitaxel formulation. Biomaterials. 2006; 27(10): 2285-2291.

21. Santra S. The potential clinical impact of quantum dots. Nanomedicine (Lond). 2012; 7(5): 623-626

22. Yong KT, Wang Y, Roy I, et al. Preparation of quantum dot/drug nanoparticle formulations for traceable targeted delivery and therapy. Theranostics. 2012; 2(7):681-694.

23. McCarthy JR Jaffer FA, Weissleder R, A macrophage-targeted theranostic nanoparticle for biomedical applications. Small. 2006; 2(8-9): 983-987.

24. Ding $\mathrm{H}, \mathrm{Wu} \mathrm{F}$. Image guided biodistribution and pharmacokinetic studies of theranostics. Theranostics. 2012; 2(11):1040-1053.

25. Velikyan I. Molecular imaging and radiotherapy: theranostics for personalized patient management. Theranostics. 2012; 2(5):424-426.

26. Choi KY, Liu G, Chen X. Theranostic nanoplatforms for simultaneous cancer imaging and therapy: current approaches and future perspectives. Nanoscale. 2012; 4(2): 330-342.

27. Win KY, Feng SS. Effect of particle size and surface coating on cellular uptake of polymeric nanoparticles for oral delivery of anticancer drugs. Biomaterials. 2005; 26(15): 2713-2722

28. Zhang S, Li J, Lykotrafitis G, et al. Size-dependent endocytosis of nanoparticles. Adv Mater. 2009. 21 (4): 419-424.

29. Decuzzi P, Ferrari M. The role of specific and non-specific interactions in receptor-mediated endocytosis of nanoparticles. Biomaterials. 2007; 28(18): 2915-2922.

30. Tan GR, Feng SS, Leong DT. The reduction of anti-cancer drug antagonism by the spatial protection of drugs with PLA-TPGS nanoparticles. Biomaterials. 2014; 35 (9): 3044-3051. 
31. Gan CW, Chien S, Feng SS. Nanomedicine: enhancement of chemotherapeutical efficacy of docetaxel by using a biodegradable nanoparticle formulation. Curr Pharm Des. 2010; 16 (21): 2308-2320.

32. Zhao J, Feng SS. Effects of PEG tethering chain length of Vitamin E TPGS with a Herceptin-functionalized nanoparticle formulation for targeted delivery of anticancer drugs. Biomaterials. 2014; 35 (10): 3340-3347.

33. Lammers T, Ulbrich K. HPMA copolymers: 30 years of advances. Adv Drug Del Rev. 2010; 62 (2): 119-121.

34. Allmeroth M, Moderegger D, Gündel D, et al. PEGylation of HPMA-based block copolymers enhances tumor accumulation in vivo: A quantitative study using radiolabeling and positron emission tomography. J Control Release. 2013; 172 (1): 77-85.

35. Kopecek J, Kopeckova P. HPMA copolymers: origins, early developments, present, and future. Adv Drug Del Rev. 2010; 62(2): 122-149.

36. Nakamura H, Etrych T, Chytil P, et al. Two step mechanisms of tumor selective delivery of $\mathrm{N}$-(2-hydroxypropyl) methacrylamide copolymer conjugated with pirarubicin via an acid-cleavable linkage. J Control Release. 2014; 174: 81-87.

37. Vasey PA, Kaye SB, Morrision R, et al. Phase I clinical and pharmacokinetic study of PK1 [N-(2-hydroxypropyl) methacrylamide copolymer doxorubicin]: first member of a new class of chemotherapeutic agents-drug-polymer conjugates. Cancer research campaign phase I/II committee. Clin Cancer Res. 1999; 5 (1): 83-94.

38. Borgman MP, Coleman T, Kolhatkar RB, et al. Tumor-targeted HPMA copolymer-(RGDfk)-(CHX-A"-DTPA) conjugates show increased kidney accumulation. J Control Release. 2008; 132(3): 193-199.

39. Yuan J, Zhang H, Kaur H, et al. Synthesis and characterization of theranostic poly(HPMA)-c(RGDyK)-DOTA-64Cu copolymer targeting tumor angiogenesis: tumor localization visualized by positron emission tomography. Mol Imaging. 2013; 12(3): 1-10

40. Mu L, Feng SS. PLGA/TPGS nanoparticles for controlled release of paclitaxel: effects of the emulsifier and drug loading ratio. Pharm Res. 2003; 20(11):1864-1872

41. Zhang Z, Tan S, Feng SS. Vitamin E TPGS as a molecular biomaterial for drug delivery. Biomaterials. 2012; 33(19): 4889-4906

42. Hu CM, Fang RH, Luk BT, et al. Polymeric nanotherapeutics: clinical development and advances in stealth functionalization strategies. Nanoscale. 2014; 6 (1): 65-75.

43. Luk BT, Fang RH, Zhang L. Lipid- and polymer-based nanostructures for cancer theranostics. Theranostics. 2012; 2(12):1117-1126.

44. Moore $\mathrm{T}$, Chen $\mathrm{H}$, Morrison $\mathrm{R}$, et al. Nanotechnologies for noninvasive measurement of drug release. Mol Pharm. 2014; 11 (1): 24-39.

45. Vijayakumar MR, Muthu MS, Singh S. Copolymers of poly(lactic acid) and D-a-tocopheryl polyethylene glycol 1000 succinate-based nanomedicines: versatile multifunctional platforms for cancer diagnosis and therapy. Expert Opin Drug Deliv. 2013; 10(4): 529-543

46. Soppimath KS, Aminabhavi TM, Kulkarni AR, et al. Biodegradable polymeric nanoparticles as drug delivery devices. J Control Release. 2001; 70 (1-2): 1-20.

47. Svenson S. Theranostics: Are we there yet? Mol Pharm. 2013; 10 (3): 848-856.

48. Mok H, Park TG. Hybrid polymeric nanomaterials for siRNA delivery and imaging. Macromol Biosci. 2012; 12 (1): 40-48.

49. Moghimi SM, Hunter AC, Murray JC. Long-circulating and target-specific nanoparticles: theory to practice. Pharmacol Rev. 2001; 53(2):283-318.

50. Gupta AK, Gupta M. Synthesis and surface engineering of iron oxide nanoparticles for biomedical applications. Biomaterials. 2005; 26 (18): 3995-4021.

51. Yen SK, Padmanabhan P, Selvan ST. Multifunctional iron oxide nanoparticles for diagnostics, therapy and macromolecule delivery. Theranostics. 2013; 3(12):986-1003

52. Li L, Jiang W, Luo K, et al. Superparamagnetic iron oxide nanoparticles as MRI contrast agents for non-invasive stem cell labeling and tracking. Theranostics. 2013; 3(8):595-615

53. Huang G, Chen H, Dong $Y$, et al. Superparamagnetic iron oxide nanoparticles: amplifying ROS stress to improve anticancer drug efficacy. Theranostics. 2013; 3(2):116-126

54. Yoo D, Lee JH, Shin TH, et al. Theranostic magnetic nanoparticles. Acc Chem Res. 2011; 44(10): 863-874

55. Clemente-Casares $X$, Santamaria P. Nanomedicine in autoimmunity. Immunol Lett. 2014; 158 (1-2): 167-174.

56. Laurent S, Forge D, Port M, et al. Magnetic iron oxide nanoparticles: synthesis, stabilization, vectorization, physicochemical characterizations, and biological applications. Chem Rev. 2008; 108 (6): 2064-2110.

57. Mornet S, Vasseur S, Fabien Grasset et al. Magnetic nanoparticle design for medical diagnosis and therapy. J Mater Chem. 2004; 14: 2161-2175.

58. Zhang $X$, Dong $Y$, Zeng $X$ et al. The effect of autophagy inhibitors on drug delivery using biodegradable polymer nanoparticles in cancer treatment. Biomaterials. 2014; 35 (6): 1932-1943.

59. Pan J, Liu Y, Feng SS. Multifunctional nanoparticles of biodegradable copolymer blend for cancer diagnosis and treatment. Nanomedicine (Lond). 2010; 5(3): 347-360.

60. Medarova Z, Pham W, Farrar C, et al. In vivo imaging of siRNA delivery and silencing in tumors. Nat Med. 2007; 13(3): 372-377.

61. Müller RH, Mäder K, Gohla S. Solid lipid nanoparticles (SLN) for controlled drug delivery - a review of the state of the art. Eur J Pharm Biopharm. 2000; 50(1): 161-177.
62. Mehnert W, Mäder K. Solid lipid nanoparticles: production, characterization and applications. Adv Drug Del Rev. 2001; 47 (2-3): 165-196.

63. Wissing SA, Kayser O, Müller RH. Solid lipid nanoparticles for parenteral drug delivery. Adv Drug Del Rev. 2004; 56 (9): 1257-1272.

64. Muller RH, Rühl D, Runge S, et al. Cytotoxicity of solid lipid nanoparticles as a function of the lipid matrix and the surfactant. Pharm Res. 1997; 14(4): 458-462.

65. Singh I, Swami R, Khan $\mathrm{W}$ et al. Lymphatic system: a prospective area for advanced targeting of particulate drug carriers. Expert Opin Drug Deliv. 2014; 11(2):211-219.

66. Shuhendler AJ, Prasad P, Leung M, et al. A novel solid lipid nanoparticle formulation for active targeting to tumor $a(v) \beta(3)$ integrin receptors reveals cyclic RGD as a double-edged sword. Adv Healthc Mater. 2012; 1(5):600-608.

67. Bae KH, Lee JY, Lee SH, et al. Optically traceable solid lipid nanoparticles loaded with siRNA and paclitaxel for synergistic chemotherapy with in situ imaging. Adv Healthc Mater. 2013; 2(4):576-584.

68. Fahmy TM, Fong PM, Park J, et al. Nanosystems for simultaneous imaging and drug delivery to T Cells. AAPS J. 2007; 9(2): E171-E180.

69. Bosman AW, Janssen HM, Meijer EW. About dendrimers: Structure, physical properties, and applications. Chem Rev. 1999; 99 (7): 1665-1688.

70. Frechet JM. Functional polymers and dendrimers - reactivity, molecular architecture, and interfacial energy. Science. 1994; 263 (5154): 1710-1715.

71. Li Y, Cheng Y, Xu T. Design, synthesis and potent pharmaceutical applications of glycodendrimers; a mini review. Curr Drug Discov Technol. 2007; 4(4): 246-254

72. Jansen JF, de Brabander-van den Berg EM, Meijer EW. Encapsulation of guest molecules into a dendritic box. Science. 1994; 266 (5188): 1226-1229

73. Grünwald GK, Vetter A, Klutz K et al. Systemic image-guided liver cancer radiovirotherapy using dendrimer-coated adenovirus encoding the sodium iodide symporter as theranostic gene. J Nucl Med. 2013; 54(8): 1450-1457.

74. Saad M, Garbuzenko OB, Ber E, et al. Receptor targeted polymers, dendrimers, liposomes: which nanocarrier is the most efficient for tumor-specific treatment and imaging? J Control Release. 2008; 130(2): 107-114.

75. Taratula O, Schumann C, Naleway MA, et al. A multifunctional theranostic platform based on phthalocyanine-loaded dendrimer for image guided drug delivery and photodynamic therapy. Mol Pharm. 2013; 10(10): 3946-3958.

76. Lasic DD. Novel applications of liposomes. Trends Biotechnol. 1998; 16(7): 307-321.

77. Torchilin VP. Recent advances with liposomes as pharmaceutical carriers. Nat Rev Drug Discov. 2005; 4(2): 145-160.

78. Voinea M, Simionescu M. Designing of 'intelligent' liposomes for efficient delivery of drugs. J Cell Mol Med. 2002; 6(4): 465-474

79. Grange C, Geninatti-Crich S, Esposito G, et al. Combined delivery and magnetic resonance imaging of neural cell adhesion molecule-targeted doxorubicin-containing liposomes in experimentally induced Kaposi's sarcoma. Cancer Res. 2010; 70(6): 2180-2190.

80. Nie Y, Ji L, Ding H, et al. Cholesterol derivatives based charged liposomes for doxorubicin delivery: preparation, in vitro and in vivo characterization. Theranostics. 2012; 2(11):1092-1103.

81. Leung SJ, Romanowski M. Light-activated content release from liposomes. Theranostics. 2012; 2(10):1020-1036.

82. Torchilin VP. Recent advances with liposomes as pharmaceutical carriers. Nat Rev Drug Discov. 2005; 4(2):145-160.

83. Papahadjopoulos D, Allen TM, Gabizon A et al. Sterically stabilized liposomes - improvements in pharmacokinetics and antitumor therapeutic efficacy. Proc Natl Acad Sci U S A. 1991; 88(24): 11460-11464.

84. Al-Jamal WT, Kostarelos K. Liposomes: From a clinically established drug delivery system to a nanoparticle platform for theranostic nanomedicine. Acc Chem Res. 2011; 44(10): 1094-1104.

85. Al-Jamal WT, Al-Jamal KT, Tian B, et al. Lipid-quantum dot bilayer vesicles enhance tumor cell uptake and retention in vitro and in vivo. ACS Nano. 2008; 2(3): 408-418.

86. Muthu MS, Feng SS. Nanopharmacology of liposomes developed for cancer therapy. Nanomedicine (Lond). 2010; 5(7): 1017-1019.

87. Muthu MS, Kulkarni SA, Xiong J et al. Vitamin E TPGS coated liposomes enhanced cellular uptake and cytotoxicity of docetaxel in brain cancer cells. Int J Pharm 2011; 421(2): 332-340.

88. Muthu MS, Kulkarni SA, Raju A, et al. Theranostic liposomes of TPGS coating for targeted co-delivery of docetaxel and quantum dots. Biomaterials. 2012; 33(12): 3494-3501.

89. Wen CJ, Zhang LW, Al-Suwayeh SA, et al. Theranostic liposomes loaded with quantum dots and apomorphine for brain targeting and bioimaging. Int $\mathrm{J}$ Nanomedicine. 2012; 7: 1599-611.

90. Wen CJ, Sung CT, Aljuffali IA, et al. Nanocomposite liposomes containing quantum dots and anticancer drugs for bioimaging and therapeutic delivery: a comparison of cationic, PEGylated and deformable liposomes. Nanotechnology. 2013; 24(32): 325101

91. Mahmud A, Xiong XB, Aliabadi HM, et al. Polymeric micelles for drug targeting. J Drug Target. 2007; 15(9): 553-584.

92. Sawant RR, Jhaveri AM, Koshkaryev A, et al. Targeted transferrin-modified polymeric micelles: enhanced efficacy in vitro and in vivo in ovarian carcinoma. Mol Pharm. 2014; 11(2): 375-381.

93. Kataoka, K, Harada, A, Nagasaki Y. Block copolymer micelles for drug delivery: design, characterization and biological significance. Adv Drug Deliv Rev. 2001; 47(1):113-131. 
94. Vriezema DM, Comellas AM, Elemans JA, et al. Self-assembled nanoreactors. Chem Rev. 2005; 105 (4): 1445-1489.

95. Kumar R, Kulkarni A, Nagesha DK, et al. In vitro evaluation of theranostic polymeric micelles for imaging and drug delivery in cancer. Theranostics. 2012; 2(7):714-722

96. Liu L, Yong KT, Roy I, et al. Bioconjugated pluronic triblock-copolymer micelle-encapsulated quantum dots for targeted imaging of cancer: in vitro and in vivo studies. Theranostics. 2012; 2(7):705-713.

97. Mi Y, Liu Y, Feng SS. Formulation of docetaxel by folic acid-conjugated d-a-tocopheryl polyethylene glycol succinate 2000 (Vitamin E TPGS(2k)) micelles for targeted and synergistic chemotherapy. Biomaterials. 2011;32(16):4058-4066

98. Torchilin VP, Lukyanov AN, Gao Z, et al. Immunomicelles: targeted pharmaceutical carriers for poorly soluble drugs. Proc Natl Acad Sci U S A. 2003; 100(10): 6039-6044.

99. Savic R, Luo LB, Eisenberg A, et al. Miceller nanocontainers distribute to defined cytoplasmic organelles. Science. 2003; 300(5619): 615-618.

100. Moghimi SM, Hunter AC, Murray JC, et al. Cellular distribution of nonionic micelles. Science. 2004; 303(5658): 626-628.

101. Kim SC, Kim DW, Shim YH, et al. In vivo evaluation of polymeric micellar paclitaxel formulation: toxicity and efficacy. J Control Release. 2001; 72(1-3):191-202.

102. Lee KS, Chung HC, Im SA, et al. Multicenter phase II trial of Genexol-PM, a Cremophor-free, polymeric micelle formulation of paclitaxel, in patients with metastatic breast cancer. Breast Cancer Res Treat. 2008; 108(2):241-250.

103. Kim DW, Kim SY, Kim HK, et al. Multicenter phase II trial of Genexol-PM, a novel Cremophor-free, polymeric micelle formulation of paclitaxel, with cisplatin in patients with advanced non-small-cell lung cancer. Ann Oncol. 2007; 108(12):2009-2014

104. Lim WT, Tan EH, Toh CK, et al. Phase I pharmacokinetic study of a weekly liposomal paclitaxel formulation (Genexol-PM) in patients with solid tumors. Ann Oncol. 2010; 21(2): 382-388.

105. Muthu MS, Kulkarni SA, Liu Y, et al. Development of docetaxel-loaded vitamin E TPGS micelles: formulation optimization, effects on brain cancer cells and biodistribution in rats. Nanomedicine (Lond). 2012; 7(3):353-364

106. Zhao J, Mi Y, Feng SS. Targeted co-delivery of docetaxel and siPlk1 by herceptin-conjugated vitamin E TPGS based immunomicelles. Biomaterials. 2013; 34(13):3411-3421.

107. Kutty RV, Feng SS. Cetuximab conjugated vitamin E TPGS micelles for targeted delivery of docetaxel for treatment of triple negative breast cancers. Biomaterials. 2013; 34(38):10160-10171.

108. Chandrasekharan P, Maity D, Yong CX, et al. Vitamin E (D-alpha-tocopheryl-co-poly(ethylene glycol) 1000 succinate) micelles-superparamagnetic iron oxide nanoparticles for enhanced thermotherapy and MRI. Biomaterials. 2011; 32(24): 5663-5672.

109. Kim KS, Park SJ, Lee MY, et al. Gold half-shell coated hyaluronic acid-doxorubicin conjugate micelles for theranostic applications. Macromol Res. 2012; 20(3): 277-282.

110. Chen H, Zhang X, Dai S, et al. Multifunctional gold nanostar conjugates for tumor imaging and combined photothermal and chemo-therapy. Theranostics. 2013; 3(9):633-49.

111. Xiao Y, Hong H, Matson VZ, et al. Gold nanorods conjugated with doxorubicin and cRGD for combined anticancer drug delivery and PET Imaging. Theranostics. 2012; 2(8):757-768.

112. Rengan AK, Jagtap M, De A, et al. Multifunctional gold coated thermo-sensitive liposomes for multimodal imaging and photo-thermal therapy of breast cancer cells. Nanoscale. 2013; 6(2):916-923.

113. Daniel MC, Astruc D. Gold nanoparticles: assembly, supramolecular chemistry, quantum-size-related properties, and applications toward biology, catalysis, and nanotechnology. Chem Rev. 2004; 104(1): 293-346.

114. Link S, El-Sayed MA. Shape and size dependence of radiative, non-radiative and photothermal properties of gold nanocrystals. Int Rev In Physical Chemistry. 2002; 19 (3): 409-453.

115. Connor EE, Mwamuka J, Gole A, et al. Gold nanoparticles are taken up by human cells but do not cause acute cytotoxicity. Small.2005; 1(3): 325-327.

116. Kumar R, Korideck $\mathrm{H}, \mathrm{Ngwa} W$, et al. Third generation gold nanoplatform optimized for radiation therapy. Transl Cancer Res. 2013; 2(4): doi: 10.3978/j.issn.2218-676X.2013.07.02

117. Han G, Martin CT, Rotello VM, et al. Stability of gold nanoparticle-bound DNA toward biological, physical, and chemical agents. Chem Biol Drug Des. 2006; 67(1): 78-82.

118. Lee SM, Kim HJ, Kim SY, et al. Drug-loaded gold plasmonic nanoparticles for treatment of multidrug resistance in cancer. Biomaterials. 2014; 35(7):2272-2282

119. Heo DN, Yang DH, Moon HJ, et al. Gold nanoparticles surface-functionalized with paclitaxel drug and biotin receptor as theranostic agents for cancer therapy. Biomaterials. 2012; 33(3): 856-866.

120. Chen $\mathrm{WH}, \mathrm{Xu} \mathrm{XD}$, Jia HZ, et al. Therapeutic nanomedicine based on dual-intelligent functionalized gold nanoparticles for cancer imaging and therapy in vivo. Biomaterials. 2013; 34(34): 8798-8807.

121. Liu Z, Liang XJ: Nano-carbons as theranostics. Theranostics.2012; 2(3): 235-237.

122. Baughman $\mathrm{RH}$ Zakhidov AA, De Heer WA Carbon nanotubes - the route toward applications. Science. 2002; 297 (5582): 787-792.

123. Fubini B, Ghiazza M, Fenoglio I. Physio-chemical features of engineered nanoparticles relevant to their toxicity. Nanotoxicology. 2010; 4: 347-363.
124. Porter AE, Gass $\mathrm{M}$, Muller $\mathrm{K}$ et al. Direct imaging of single-walled carbon nanotubes in cells. Nat Nanotechnol. 2007; 2:713 - 717

125. McDevitt MR, Chattopadhyay D, Kappel BJ, et al. Tumor targeting with antibody-functionalized, radiolabeled carbon nanotubes. J Nuclear Med. 2007; 48(7): 1180-1189.

126. Yang K, Feng $L$, Shi $X$, et al. Nano-graphene in biomedicine: theranostic applications. Chem Soc Rev. 2013; 42(2): 530-547.

127. Shen H, Zhang L, Liu M, et al. Biomedical applications of graphene. Theranostics. 2012; 2(3):283-294

128. Chen Z, Ma L, Liu Y et al. Applications of functionalized fullerenes in tumor theranostics. Theranostics. 2012; 2(3):238-250.

129. Nerl HC, Cheng C, Goode AE, et al. Imaging methods for determining uptake and toxicity of carbon nanotubes in vitro and in vivo. Nanomedicine (Lond). 2011; 6 (5): 849-865.

130. Boncel S, Müller KH, Skepper JN et al. Tunable chemistry and morphology of multi-wall carbon nanotubes as a route to non-toxic, theranostic systems. Biomaterials. 2011; 32 (30): 7677-7686.

131. Robinson JT, Welsher K, Tabakman SM, et al. High performance in vivo near-IR $(>1 \mu \mathrm{m})$ imaging and photothermal cancer therapy with carbon nanotubes. Nano Res. 2010; 3 (11): 779-793.

132. Das M, Datir SR, Singh RP, et al. Augmented anticancer activity of a targeted, intracellularly activatable, theranostic nanomedicine based a fluorescent and radiolabeled, methotrxate-folic acid-multiwalled carbon nanotube conjugate. Mol Pharm. 2013; 10 (7): 2543-2557.

133. Qin SY, Feng J, Rong L, et al. Theranostic GO-based nanohybrid for tumor induced imaging and potential combinational tumor therapy. Small. 2014; 10 (3): 599-608.

134. Muthu MS, Wilson B. Multifunctional radionanomedicine: a novel nanoplatform for cancer imaging and therapy. Nanomedicine (Lond). 2010; 5(2):169-171.

135. Bardhan R, Lal S, Joshi A, et al. Theranostic nanoshells: From probe design to imaging and treatment of cancer. Acc Chem Res. 2011; 44(10):936-946.

136. Muthu MS, Feng SS. Targeted nanomedicine for detection and treatment of circulating tumor cells. Nanomedicine (Lond). 2011; 6(4):579-581.

137. Ho D, Sun X, Sun S. Monodisperse magnetic nanoparticles for theranostic applications. Acc Chem Res. 2011; 44(10): 875-882.

138. Zhu Y, Hong H, Xu ZP, et al. Quantum dot-based nanoprobes for in vivo targeted imaging. Curr Mol Med. 2013; 13(10):1549-1567.

139. Wu X, Tian F, Zhao JX, et al. Evaluating pharmacokinetics and toxicity of luminescent quantum dots. Expert Opin Drug Metab Toxicol. 2013; 9(10): 1265-1277.

140. Tsukahara T, Haniu H. Cellular cytotoxic response induced by highly purified multi-wall carbon nanotubes in human lung cells. Mol Cell Biochem. 2011; 352(1-2): 57-63.

141. Yamashita K, Yoshioka Y, Higashisaka K,et al. Carbon nanotubes elicit DNA damage and inflammatory response relative to their size and shape. Inflammation. 2010; 33: 276-280.

142. Ge C, Meng L, Xu L,et al. Acute pulmonary and moderate cardiovascular responses of spontaneously hypertensive rats after exposure to single-wall carbon nanotubes. Nanotoxicology. 2012; 6(5): 526-542.

143. Muthu MS. Abdulla A, Pandey BL. Major toxicities of carbon nanotubes induced by reactive oxygen species: should we worry about the effects on the lungs, liver and normal cells? Nanomedicine (Lond). 2013; 8(6): 863-866.

144. Yang ST, Luo J, Zhou Q, et al. Pharmacokinetics, metabolism and toxicity of carbon nanotubes for biomedical purposes. Theranostics. 2012; 2(3):271-282

145. Mateo D, Morales P, Avalos A, et al. Oxidative stress contributes to gold nanoparticle-induced cytotoxicity in human tumor cells. Toxicol Mech Methods. 2014; 24(3): 161-172.

146. Yuan X, Setyawati MI, Leong DT, et al. Ultrasmall $\mathrm{Ag}^{+}$-rich nanoclusters as highly efficient nanoreservoirs for bacterial killing. Nano Res. 2013; doi: 10.1007/s12274-013-0395-6. [Epub ahead of print].

147. Setyawati MI, Tay CY, Leong DT. Effect of zinc oxide nanomaterials-induced oxidative stress on the p53 pathway. Biomaterials. 2013; 34(38): 10133-10142.

148. Tay CY, Fang W, Setyawati MI, et al. Reciprocal response of human oral epithelial cells to internalized silica nanoparticles. Part Part Syst Charact. 2013; 30(9): 784-793.

149. Setyawati MI, Fang WR, Chia SL, et al. Nanotoxicology of common metal oxide based nanomaterials: their ROS-y and non-ROS-y consequences. Asia-Pacific J Chem Eng. 2013; 8(2):205-217.

150. Setyawati MI, Khoo PK, Eng BH, et al. Cytotoxic and genotoxic characterization of titanium Dioxide, gadolinium oxide and poly(lactic-co-glycolic acid) nanoparticles in human fibroblasts. J Biomed Mat Res Part A. 2013; 101(3):633-640.

151. Ng KW, Khoo PKS, Heng BC, et al. Cellular DNA damage response to zinc oxide nanoparticles hinges on the tumor suppressor p53 pathway. Biomaterials. 2011:32(32):8218-8225.

152. Setyawati MI, Tay CY, Chia SL, et al. $\mathrm{TiO}_{2}$ nanomaterials cause endothelial cell leakiness by disrupting the homophilic interaction of VE-cadherin. Nat Commun. 2013; 4:1673, doi: 10.1038/ncomms2655. [Epub ahead of print].

153. Tay CY, Cai PQ, Setyawati MI, et al. Nanoparticles strengthen intracellular tension and retard cellular migration. Nano Lett. 2014; 14(1): 83-88. 


\section{Author Biography}

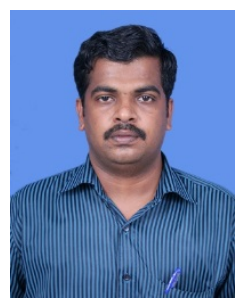

Dr Madaswamy S. Muthu earned Bachelor degree in Pharmacy, Master's in Pharmaceutical Technology from India and Ph.D. degree in Pharmaceutics from IIT, Varanasi, India in 2009. He did his postdoctoral training in Department of Chemical Engineering at National University of Singapore as a recipient of BOYSCAST Fellowship and CREST Award from India. Muthu's research interest is to develop nanomedicine as multi-functional platform for diagnosis and therapy. He has authored over 35 peer-reviewed publications with a cumulative impact factor of $>110$ and h-Index of 12 .

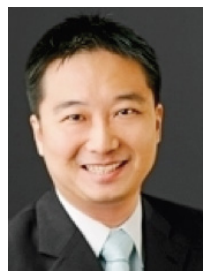

Dr David Tai Leong's research focuses on understanding nanomaterials interactions with biological systems, translating this understanding to useful biomaterials and using bio-derived materials in interesting non-biological applications. His work has been published in top journals like Nature Communications, Nano Letters, NPG Asia Materials and Biomaterials. He graduated from National University of Singapore with a Bachelor of Chemical Engineering and a PhD in Biology. He did his postdoctoral training at the Howard Hughes Medical Institute at University of Pennsylvania.

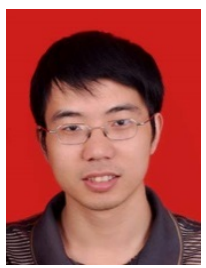

Dr Lin Mei is an Associate Professor at Tsinghua University Shenzhen Graduate School in biomaterials, drug delivery and nanomedicine. He got his bachelor's degree from Fudan University, and PhD from Peking Union Medical College, Tsinghua University. He has co-authored over 40 SCI articles that resulted in 15 citations per article and h-Index 13.

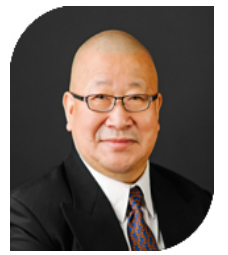

Dr Si-Shen Feng is a Professor in biomaterials and nanomedicine. He got his 6-year diploma from Peking University, MEng from Tsinghua University and $\mathrm{PhD}$ from Columbia University. He has co-authored over 140 WOS articles that resulted in 35 citations per article and h-Index 39. Currently he is an Associate Editor of Biomaterials (2012 JIF=7.604) and Nanomedicine (2012 JIF=5.260). His research interest includes 1) Molecular biomaterials, 2) nanomedicine for drug targeting. 3) nanomedicine for multimodality cancer therapy, 4) si-RNA based nanomedicine; 6) nanoimmunotherapy and 7) nanomedicine for treatment of cancer stem cells etc. 